Comment. Math. Helv. 86 (2011), 609-657

DOI $10.4171 / \mathrm{CMH} / 236$
Commentarii Mathematici Helvetici

(C) Swiss Mathematical Society

\title{
Modules for $\mathbb{Z} / p \times \mathbb{Z} / p$
}

Jon F. Carlson, Eric M. Friedlander ${ }^{* *}$ and Andrei Suslin ${ }^{* * *}$

\begin{abstract}
We investigate various aspects of the modular representation theory of $\mathbb{Z} / p \times \mathbb{Z} / p$ with particular focus on modules of constant Jordan type. The special modules we consider and the constructions we introduce not only reveal some of the structure of $(\mathbb{Z} / p \times \mathbb{Z} / p)$-modules but also provide a guide to further study of the representation theory of more general finite group schemes.
\end{abstract}

Mathematics Subject Classification (2010). 16G10, 20C20, $20 \mathrm{G} 10$.

Keywords. Modular representations, Jordan type.

\section{Introduction}

In this paper, we investigate finite dimensional $k G$-modules, where $k$ is a field of characteristic $p>0$ and $G=\mathbb{Z} / p \times \mathbb{Z} / p$. Our objective is to introduce some new constructions and invariants for modules and to explore some of their properties. For $p>2, k G$ has wild representation type so that this elementary group algebra provides a good test for various constructions and techniques in modular representation theory. For example, the special case of $G=\mathbb{Z} / p \times \mathbb{Z} / p$ proved to be critical in the proof given in [7] that the generic and maximal Jordan types are well defined for a finite dimensional module over an arbitrary finite group scheme.

In joint work with Julia Pevtsova [2], we introduced a special class of modules for finite groups schemes, those of constant Jordan type. Most of the $k G$-modules we consider in this paper satisfy this property of constant Jordan type, and much of our effort is directed toward understanding as much as possible for such modules. We introduce a more restrictive property, the "equal images property", satisfied by certain $k G$-modules and closed under taking quotients. We observe in Proposition 5.8 that there is a vast array of non-isomorphic indecomposable $k G$-modules which satisfy this equal images property, suggesting that $k G$-modules of constant Jordan type constitute a wild category for $p>2$.

\footnotetext{
*Partially supported by the NSF grant \# 0654173.

*** Partially supported by the NSF grant \# 0300525.

**** Partially supported by the NSF.
} 
Our examples and constructions not only reveal interesting modules for $k G$ but also suggest possible extensions from $G$ to more general finite group schemes.

We begin with the equal images property and a particularly explicit class of $k G$ modules satisfying the property. These we call " $W$ modules". $W$ modules of the form $W_{n, 2}$ have appeared in earlier work as "zig-zag" modules. Despite their seemingly special nature, the class of $W$ modules provides a splitting of the Jordan type functor from the Grothendieck group of modules of constant Jordan type to $\mathbb{Z}^{p}$ (Theorem 4.2). Moreover, every $k G$-module with the equal images property is a quotient of a $W$ module (Theorem 5.4).

In [6], the second author and Julia Pevtsova introduced vector bundles associated to finite dimensional modules for an infinitesimal group scheme which have constant $j$-type. This construction of vector bundles holds considerable promise in that it distinguishes various modules with the same local Jordan type and might exhibit explicit newly discovered vector bundles on quite elaborate singular varieties. In the special case of the infinitesimal group scheme $\mathbb{G}_{a(1)}^{2}$ with group algebra isomorphic to $k G$, this construction produces vector bundles on $\mathbb{P}^{1}(k)$ which are accessible to computation. We give explicit determination of the first and second kernel bundles for $W$ modules.

We introduce the construction of the "generic kernel" (as well as the "generic image") of a finite dimensional $k G$-module, a module which has the equal image property and thus is of constant Jordan type. This is essentially an extension of a construction introduced in [7] in order to prove that the "maximal Jordan type" of a $k G$-module is well defined, independent of the choice of generators of the augmentation ideal of $k G$. The generic kernel of a finite dimensional $k G$-module $M$ can be characterized as the maximal submodule of $M$ which has the equal images property (Proposition 7.8).

In Theorem 8.10 we exhibit an interesting filtration on $k G$-modules of constant rank which differs from the radical filtration. This filtration involves duality relating generic kernels and generic images, providing further insight into specific $k G$-modules. A cautionary example is provided in Example 9.4.

Corollary 11.3 identifies all cyclic $k G$-modules of constant Jordan type: they are all of the form $k G / I^{t}$, a quotient of $k G$ by some power of the augmentation ideal. This result is surprisingly difficult to prove, and requires properties of the Wronskian of a collection of polynomials with coefficients in a field of characteristic $p>0$.

We conclude this paper by identifying various vector bundles over $\mathbb{P}^{1}$ associated with certain classes of $k G$-modules. For this, we invoke a basic property of generic kernels to determine kernel bundles for cyclic $k G$-modules of constant Jordan type. We also determine kernel bundles for Heller shifts of the trivial module and certain $k G$-modules of constant rank associated to nilpotent cohomology classes.

We reiterate that unless specified otherwise $p$ denotes a prime number, $k$ is a field of characteristic $p$, and $G$ denotes the group $\mathbb{Z} / p \times \mathbb{Z} / p$. If $M$ is a vector space over 
$k$ (perhaps with more structure, such as the structure of a $k G$-module) and if $K / k$ is a field extension, then we denote by $M_{K}$ the $K$-vector space $K \otimes_{k} M$. For notational simplicity, we shall denote $M \otimes_{k} N$ by $M \otimes N$ for vector spaces $M, N$ over $k$.

We gratefully acknowledge numerous useful conversations with Julia Pevtsova. We also thank the Mathematical Sciences Research Institute for its hospitality during the spring of 2008 when some of this paper was written. Furthermore, we thank the referee for a very detailed reading of this paper.

\section{Recollections}

For the convenience of the reader, we briefly summarize here some of the general results to be found in earlier papers which serve as the foundations for this present work. These general results are typically formulated for an arbitrary finite group scheme over $k$, our "base field" of characteristic $p>0$. Some results only apply to infinitesimal group schemes. Since the group algebra of $\mathbb{Z} / p \times \mathbb{Z} / p$ is isomorphic to the group algebra of the infinitesimal group scheme $\mathbb{G}_{a(1)} \times \mathbb{G}_{a(1)}$, these results are fully available in our study of $k \mathbb{Z} / p \times \mathbb{Z} / p$-modules.

The isomorphism type of a finite dimensional $k[t] /\left(t^{p}\right)$-module $M$ is said to be the Jordan type of $M$. For $M$ of dimension $m$, this Jordan type is specified by a partition of $m$; the Jordan types are partially ordered according to the dominance ordering of partitions. We denote a Jordan type by JType $(M)$. Should we wish to be more explicit, we denote the Jordan type of $M$ by $\sum_{i=1}^{p} a_{i}[i]$ with $m=\sum_{i=1}^{p} a_{i} i$, meaning that the Jordan canonical form of $t$ on $M$ has $a_{i}$ blocks of size $i$. Thus. the indecomposable $k[t] /\left(t^{p}\right)$-modules of dimension $i$ has Jordan type $[i]$.

For an arbitrary finite group scheme $G$, the cohomological variety, Spec $\mathrm{H}^{\bullet}(G, k)$, is the prime ideal spectrum of the finitely generated commutative $k$-algebra $\mathrm{H}^{\bullet}(G, k)$, where $\mathrm{H}^{\bullet}(G, k) \subset \mathrm{H}^{*}(G, k)$ is the subalgebra of the cohomology algebra $\mathrm{H}^{*}(G, k)$ generated by the elements of even degree if $p$ is odd, and $\mathrm{H}^{\bullet}(G, k)$ is the full algebra $\mathrm{H}^{*}(G, k)$ if $k$ has characteristic 2. If $M$ is a $k G$-module, then the cohomological support variety of $M$ is the spectrum of the quotient of $\mathrm{H}^{\bullet}(G, k)$ by the annihilator ideal of $\operatorname{Ext}_{k G}^{*}(M, M)$,

$$
\operatorname{Spec} \mathrm{H}^{\bullet}(G, k) / \operatorname{Ann}_{\mathrm{H}^{\bullet}(G, k)}\left(\operatorname{Ext}_{k G}^{*}(M, M)\right) \text {. }
$$

It is convenient to view support varieties in terms of $\pi$-points of $G$.

Definition 1.1. Let $G$ be an elementary abelian $p$-group. A $\pi$-point $\alpha_{K}: K[t] /\left(t^{p}\right) \rightarrow$ $K G$ is a flat map of $K$-algebras for some field extension $K / k$. (If the group $G$ were not a $p$-group, then the definition would also impose the condition that $\alpha_{K}$ factor through a unipotent subgroup scheme.) 
Two $\pi$-points, $\alpha_{K}: K[t] /\left(t^{p}\right) \rightarrow K G$ and $\beta_{L}: L[t] /\left(t^{p}\right) \rightarrow L G$ are equivalent if for any finite dimensional $k G$-module $M$, the restriction of $M_{K}=K \otimes M$ along $\alpha_{K}$ is a free $K G$-module if and only if the restriction of $M_{L}$ along $\beta_{L}$ is a free $L G$-module.

The set of equivalence classes of $\pi$-points of $G$ is the set of points of a projective $k$-scheme $\Pi(G)$.

The $\Pi$-support space of a $k G$-module $M$ is the reduced subscheme $\Pi(G)_{M} \subset$ $\Pi(G)$ whose points are those represented by $\pi$-points $\alpha_{K}: K[t] /\left(t^{p}\right) \rightarrow K G$ with the property that $\alpha_{K}^{*}\left(M_{K}\right)$ is not projective.

As verified in [5], Theorem 7.5, for any finite group scheme $G$ there is a natural isomorphism $\operatorname{Proj} \mathrm{H}^{\bullet}(G, k) \cong \Pi(G)$ which restricts to an isomorphism

$$
\operatorname{Proj}\left(\mathrm{H}^{\bullet}(G, k) / \operatorname{Ann}_{\mathrm{H}^{\bullet}(G, k)}\left(\operatorname{Ext}_{k G}^{*}(M, M)\right)\right) \cong \Pi(G)_{M} .
$$

Definition 1.2. A finite dimensional $k G$-module $M$ is said to have constant rank if the rank of the operator $\alpha_{K}(t): M_{K} \rightarrow M_{K}$ is independent of choice of the $\pi$-point $\alpha_{K}: K[t] /\left(t^{p}\right) \rightarrow K G$.

Similarly, $M$ is said to have constant $j$-rank (for some $j, 1 \leq j<p$ ) if the rank of $\left(\alpha_{K}(t)\right)^{j}: M_{K} \rightarrow M_{K}$ is independent of choice of $\pi$-point $\alpha_{K}: K[t] /\left(t^{p}\right) \rightarrow K G$.

Finally, $M$ is said to have constant Jordan type if the Jordan type of $\alpha_{K}(t): M_{K} \rightarrow$ $M_{K}$ is independent of choice of $\pi$-point $\alpha_{K}: K[t] /\left(t^{p}\right) \rightarrow K G$.

For infinitesimal group schemes, there is yet another perspective on support varieties.

Definition 1.3. Let $G$ be an infinitesimal group scheme over $k$. A 1-parameter subgroup of $G$ of height $r$ over a field extension $K / k$ is a morphism $\mu_{K}: \mathbb{G}_{a(r), K} \rightarrow G_{K}$ of group schemes over $K$. There is a naturally constructed affine group scheme $V_{r}(G)$ whose points with values in any commutative $k$-algebra $A$ are naturally identified with the set of morphisms $\mu: \mathbb{G}_{a(r), A} \rightarrow G_{A}$ of group schemes over $A$.

As verified in [13], Theorem 5.2 and Corollary 6.8, there is a natural morphism $V_{r}(G) \rightarrow \operatorname{Spec~}^{\bullet}(G, k)$ which is a homeomorphism of underlying spaces for any infinitesimal group scheme of height $\leq r$; moreover, this homeomorphism restricts to a homeomorphism of closed subspaces

$$
V_{r}(G)_{M} \cong \operatorname{Spec~}^{\bullet}(G, k) / \operatorname{Ann}_{\mathrm{H}^{\bullet}(G, k)}\left(\operatorname{Ext}_{k G}^{*}(M, M)\right),
$$

where $V_{r}(G)_{M} \subset V_{r}(G)$ is the closed subvariety consisting of those 1-parameter subgroups $\mu_{K}: \mathbb{G}_{a(r)_{K}} \rightarrow G_{K}$ such that $\left(\mu_{K} \circ \epsilon\right)^{*}\left(M_{K}\right)$ is not projective. Here, $\epsilon: k[t] /\left(t^{p}\right) \rightarrow k G_{a(r)}$ is the map of algebras (but not of Hopf algebras) sending $t$ to the divided power operator $\left.\left(\frac{d}{d t}\right)^{(r-1)}\right)$. 
For $G=\mathbb{Z} / p^{\times r}$, an elementary abelian $p$-group of rank $r$, we have isomorphisms of $k$-schemes

$$
\begin{gathered}
\mathbb{A}^{r} \cong \operatorname{Spec} \mathrm{H}^{\bullet}(G, k) \cong V_{1}\left(\mathbb{G}_{a(1)}^{\times r}\right), \\
\mathbb{P}^{r-1} \cong \operatorname{Proj} \mathrm{H}^{\bullet}(G, k) \cong \Pi(G) .
\end{gathered}
$$

For any scheme $X$ over $k$ and any commutative $k$-algebra $A$ we denote by $X(A)$ the set of $A$-valued maps Spec $A \rightarrow X$. In particular, $\mathbb{P}^{1}(k)$ denotes the set of $k$-rational points of $\mathbb{P}^{1}$.

Finally, we recall the structure of algebraic vector bundles on $\mathbb{P}^{1}$ given by A. Grothendieck in [8], Theorem 1. Any such bundle is a direct sum of the line bundles of the form $\mathcal{O}_{\mathbb{P}^{1}}(n)$ for some $n \in \mathbb{Z}$, the Serre twists of the structure sheaf $\mathcal{O}_{\mathbb{P}^{1}}$. Under Serre's correspondence (see [9], II.5.15), $\mathcal{O}_{\mathbb{P}}(n)$ corresponds to the graded module $A[n]$ for the graded ring $A=k[x, y]$, where $A[n]$ in degree $d$ equals $A$ in degree $d+n$.

\section{The equal images property}

Although this paper concerns the representation theory of $\mathbb{Z} / p \times \mathbb{Z} / p$, this section is valid for an elementary abelian $p$-group of arbitrary rank. Thus, in this section, we take $G=(\mathbb{Z} / p)^{r}$ for some $r \geq 2$. Our purpose in this section is to introduce the "equal images property" for a finite dimensional $k G$-module and to explore some of its implications.

For notation, we let $k G=k\left[t_{1}, \ldots, t_{r}\right] /\left(t_{1}^{p}, \ldots, t_{r}^{p}\right)$. The radical of $k G$ is the augmentation ideal $\operatorname{Rad}(k G)$ generated by $x_{1}, \ldots, x_{r}$ where for each $i, x_{i}$ is the class of $t_{i}$ modulo the ideal $\left(t_{1}^{p}, \ldots, t_{r}^{p}\right)$. Thus, $x_{i}^{p}=0$ for all $i$.

The data of a $K$-algebra homomorphism $\alpha_{K}: K[t] /\left(t^{p}\right) \rightarrow K G$ is equivalent to a choice of

$$
\ell_{\alpha_{K}}=\alpha_{K}(t) \in K G ;
$$

the condition that $\alpha_{K}$ be flat is equivalent to the condition that $\ell_{\alpha_{K}}$ be an element of $\operatorname{Rad}(K G) \backslash \operatorname{Rad}^{2}(K G)$, that is, in the radical of $K G$ (since $t \in K[t] /\left(t^{p}\right)$ is nilpotent) but not in the square of the radical of $K G$. In what follows we let $\ell_{\alpha_{K}}=\ell_{\alpha}$, the field $K$ being implicit in the definition of $\alpha$.

The condition that the finite dimensional $k G$-module $M$ has constant Jordan type can be reformulated in more classical terms as follows.

Proposition 2.1. A finite dimensional $k G$-module $M$ has constant Jordan type if and only if for some algebraic closure $K / k$ and for all non-zero $r$-tuples $0 \neq \underline{a}=$ $\left(a_{1}, \ldots, a_{r}\right) \in K^{r}$, the Jordan type of $\sum_{i} a_{i} x_{i}$ acting on $M_{K}$ is independent of $\underline{a}$. 
Proof. Let $K$ be an algebraic closure of $k$. For $0 \neq \underline{a}=\left(a_{1}, \ldots, a_{r}\right) \in K^{r}$, let $\alpha_{\underline{a}}: K[t] /\left(t^{p}\right) \rightarrow K G$ be the $\pi$-point which takes $t$ to $\sum a_{i} t_{i}$. Clearly, if $M$ has constant Jordan type, then that type is the Jordan type at every $\alpha_{a}$.

We recall that there is a partial ordering on Jordan types of $\pi$-points on $M$ given by the dominance ordering on partitions of the dimension of $M$. We use [7], 2.7, which asserts the following two non-trivial properties of maximal and generic Jordan types. First, the Jordan type of $M$ at any $\pi$-point whose equivalence class in $\Pi(G)=\mathbb{P}^{r-1}$ is a generic point, is greater or equal to the Jordan type at any $\pi$-point of $k G$. Second, if $\alpha_{K}^{*}\left(M_{K}\right)$ achieves this maximal Jordan type (i.e., the generic Jordan type), then this maximal Jordan type is also the Jordan type for any $\beta_{L}^{*}\left(M_{L}\right)$ whenever $\alpha_{K}$ is equivalent to $\beta_{L}$.

To prove the converse, we now assume that $\alpha_{a}^{*}\left(M_{K}\right)$ has Jordan type independent of $0 \neq \underline{a} \in K^{r}$ for some $K / k$ algebraically closed. Then for an explicit choice of representative of the generic point of $\Pi(G)$ the Jordan type is the same as that of each $\alpha_{a}^{*}\left(M_{K}\right)$ (using the fact that maximality of the Jordan type of $M$ for points of $\mathbb{A}^{r}$ is an open condition). Thus, this common Jordan type is the maximal Jordan type of $M$ among all $\pi$-points. Because maximality of the Jordan type of $M$ is achieved by some representative of a point of $\Pi(G)$ if and only if it is achieved at every representative, maximality of the Jordan type of $M$ is an open condition on the scheme of $\Pi(G)$ of equivalence classes of $\pi$-points. Because the points of $\Pi(G)$ represented by $\pi$-points of the form $\alpha_{a}$ are dense by the Hilbert Nullstellensatz, we conclude that maximality holds at every $\pi$-point of $k G$. In other words, $M$ must have constant Jordan type.

In much of what follows we take some care to avoid imposing any condition other than the characteristic on our base field $k$. Some of the reasons for this care are revealed in the following cautionary example.

Example 2.2. Let $G=\mathbb{Z} / p \times \mathbb{Z} / p$ and suppose that $k$ is not algebraically closed. Write $k G=k[x, y] /\left(x^{p}, y^{p}\right)$. Let $f(z)$ be a polynomial of degree $n$ which has no root in $k$. Consider the $2 n$-dimensional $k G$-module with $x, y$ acting as

$$
x \rightarrow\left(\begin{array}{cc}
0 & 0 \\
I_{n} & 0
\end{array}\right), \quad y \rightarrow\left(\begin{array}{cc}
0 & 0 \\
C_{n} & 0
\end{array}\right) .
$$

where $I_{n}$ is the $n \times n$ identity matrix and $C_{n}$ is the companion matrix of $f(z)$ (so that $\left.f(z)=\operatorname{Det}\left(z I_{n}-C_{n}\right)\right)$.

Then for any $0 \neq(a, b) \in k^{2}$, the image of $a x+b y$ on $M$ has dimension $n$, equal to $\operatorname{Rad}(M)$. This easily implies that the image of $a x+b y$ on $M$ is independent of $0 \neq(a, b) \in k^{2}$. Thus, the Jordan type of $M$ at $k$-rational points of $\mathbb{A}^{2}$ is independent of the choice of $k$-rational point, and in fact equals the maximal Jordan type of $M$. However, if we take $K / k$ to be a finite field extension in which $f(x)$ has a root $\gamma$, 
then the rank of $\gamma x-y: M_{K} \rightarrow M_{K}$ has dimension less than $n$. Hence, $M$ does not have constant Jordan type or constant rank.

We now introduce a property of certain $k G$-modules which we shall see implies the property of constant Jordan type.

Definition 2.3. A finite dimensional $k G$-module $M$ is said to have the equal images property if

$$
\left(\ell_{\alpha}\left(M_{K}\right)\right)_{\Omega}=\left(\ell_{\beta}\left(M_{L}\right)\right)_{\Omega}
$$

for any two $\pi$-points $\alpha_{K}: K[t] /\left(t^{p}\right) \rightarrow K G$ and $\beta_{L}: L[t] /\left(t^{p}\right) \rightarrow L G$ and for any field extension $\Omega$ of both $K, L$. Here, as in (1), $\ell_{\alpha}=\alpha_{K}(t)$.

Our notation here is that

$$
\ell_{\alpha}\left(M_{K}\right)=\operatorname{Im}\left\{\ell: M_{K} \rightarrow M_{K}\right\}
$$

is the image of multiplication by $\ell_{\alpha}$ on $M_{K}$. Observe that in Definition 2.3 it suffices to consider fields $K, L, \Omega$ which are finitely generated over $k$.

The equal images property was called the "constant images property" in [1].

We shall need some equivalent conditions for the equal images property. The following lemma will be useful for this purpose. Here we make no assumptions on the field $k$ other than that it has characteristic $p$.

Lemma 2.4. Let $\ell$ be any element which is in $\operatorname{Rad}(k G)$ but not in $\operatorname{Rad}^{2}(k G)$. We write $\ell=\sum_{i=1}^{r} a_{i} x_{i}+u$ for $a_{1}, \ldots, a_{r} \in k$ and $u \in \operatorname{Rad}^{2}(k G)$. Then for any $k G$-module $M$ it must be that $\ell M=\operatorname{Rad}(M)$ if and only if $\left(\sum_{i=1}^{r} a_{i} x_{i}\right) M=\operatorname{Rad}(M)$.

Proof. Let $N=\operatorname{Rad}(M)$ and suppose that $\ell M=\operatorname{Rad}(M)=N$. So $u M \subseteq$ $\operatorname{Rad}(N)$, and by Nakayama's Lemma [3], we have that $\left(\sum_{i=1}^{r} a_{i} x_{i}\right) M=N=$ $\operatorname{Rad}(M)$. This proves one direction. The proof in the other direction is almost identical.

With the above lemma, we can give an equivalent definition for the equal images property.

Proposition 2.5. Suppose that $M$ is a $k G$-module. The following are equivalent.

(1) The module $M$ has the equal images property.

(2) For any extension $K$ of $k$ and any $r$-tuple $\underline{a}=\left(a_{1}, \ldots, a_{r}\right) \in K^{r}$ of elements of $K$, such that not all of the $a_{i}$ 's are zero, we have that $\left(\sum_{i=1}^{r} a_{i} x_{i}\right) M_{K}=$ $\operatorname{Rad}\left(M_{K}\right)$. 
Proof. First suppose that $M$ has the equal images property. Then for all $i$ and $j$ we have that $x_{i} M=x_{j} M$ and hence that $x_{i} M=\sum_{j=1}^{r}\left(x_{j} M\right)=\operatorname{Rad}(M)$. Now suppose that $K$ is any extension of $k$ and that $\alpha_{K}: K[t] /\left(t^{p}\right) \rightarrow k G$ is the $\pi$-point given by $\alpha_{K}(t)=\sum_{i=1}^{r} a_{i} x_{i}$. Then by the equal images property

$$
\ell_{\alpha}\left(M_{K}\right)=x_{1} M_{K}=x_{1}(K \otimes M)=K \otimes x_{1} M=K \otimes \operatorname{Rad}(M)=\operatorname{Rad}\left(M_{K}\right) .
$$

This shows that (1) implies (2). The reverse implication is straightforward with the help of Lemma 2.4.

A main point of the next proposition is that everything becomes much easier when the field of coefficients is algebraically closed.

Proposition 2.6. Let $M$ be a finite dimensional $k G$-module and let $K$ be an algebraic closure of $k$. Then the following are equivalent:

(1) $M$ has the equal images property.

(2) For all $\ell \in \operatorname{Rad}(K G) \backslash \operatorname{Rad}^{2}(K G)$, we have that $\ell\left(M_{K}\right)=\operatorname{Rad}\left(M_{K}\right)$.

(3) The submodule $\ell\left(M_{K}\right) \subset M_{K}$ is independent of $\ell \in \operatorname{Rad}(K G) \backslash \operatorname{Rad}^{2}(K G)$.

(4) For all $0 \neq \underline{a} \in K^{r}, \operatorname{Im}\left\{\sum_{i=1}^{r} a_{i} x_{i}: M_{K} \rightarrow M_{K}\right\}=\operatorname{Rad}\left(M_{K}\right)$.

(5) The submodule $\left(\sum_{i=1}^{r} a_{i} x_{i}\right)\left(M_{K}\right) \subset M_{K}$ is independent of the non-zero element $\underline{a}=\left(a_{1}, \ldots, a_{r}\right) \in K^{r}$.

Proof. The implication (1) $\Longrightarrow(2)$ is a consequence of Lemma 2.4 and Proposition 2.5. Also, (2) $\Longleftrightarrow$ (4), by Lemma 2.4. It is obvious that (2) $\Longrightarrow(3) \Longleftrightarrow$ (5). If (5) holds, then we must have that $x_{i} M_{K}=x_{j} M_{K}$ for all $i$ and $j$. Therefore, for any $0 \neq \underline{a} \in K^{r}$, we have that $\operatorname{Im}\left\{\sum_{i=1}^{r} a_{i} t_{i}: M_{K} \rightarrow M_{K}\right\}=x_{1} M_{K}=\operatorname{Rad}\left(M_{K}\right)$. Hence, (5) $\Longleftrightarrow$ (4). It remains only to show that (1) is implied by any of the other statements.

To prove that (2) $\Longleftrightarrow(1)$, it suffices to assume (2) and consider some flat map $\beta_{L}: L[t] /\left(t^{p}\right) \rightarrow L G$ with $L / K$ finitely generated, then prove that $\beta_{L}\left(M_{L}\right)=$ $\operatorname{Rad}\left(M_{L}\right)$. We proceed by contradiction. Assume that $\ell_{\beta}\left(M_{L}\right) \neq \operatorname{Rad}\left(M_{L}\right)$, so that the dimension $\ell_{\beta}\left(M_{L}\right)$ is strictly less than the dimension of $\operatorname{Rad}\left(M_{L}\right)$. Choose a finitely generated $K$-subalgebra $A \subset L$ with field of fractions $L$ such that there exists some flat $A$-algebra homomorphism $\beta_{A}: A[t] /\left(t^{p}\right) \rightarrow A G$ with the property that $\beta_{L}=L \otimes_{A} \beta_{A}$. For some specialization $\phi: A \rightarrow K$ determining $\alpha_{K}=$ $K \otimes_{A} \beta_{A}: K[t] /\left(t^{p}\right) \rightarrow K G$, the dimension of $\ell_{\alpha}\left(M_{K}\right)$ (which is equal to the specialization of the Noetherian $A$-module $\left(\beta_{A}(t)\left(M_{A}\right)\right.$ at $\left.\phi\right)$ is strictly less than the dimension of $\operatorname{Rad}\left(M_{K}\right)$. This contradicts (2).

We give the following first example of a $k G$-module with the equal images property. 
Example 2.7. Let $I=\operatorname{Rad}(k G) \subset k G$ be the augmentation ideal. Then $I^{j}$ has constant Jordan type for all $j, 0 \leq j \leq r p-1$ (and $I^{r p}=0$ ). This is a consequence of the fact that $I$ is invariant under all automorphisms of the group algebra and whenever $u, v$ are two elements of $\operatorname{Rad}(k G)$ that are not in $\operatorname{Rad}^{2}(k G)$, then there is an automorphism that takes $u$ to $v$. On the other hand, $I^{j}$ has the equal images property if and only if $(r-1)(p-1) \leq j$. That is, for $j<(r-1)(p-1)$, it is straightforward to construct a monomial in $x_{1}, \ldots, x_{r}$ that is a multiple of $x_{1}$ but not a multiple of $x_{2}$. (See Example 3.2 below.)

The preceding example suggests the following relationship between the constant Jordan type property and the equal images property.

Proposition 2.8. Let $M$ be a finite dimensional $k G$-module with the equal images property. Then

(1) $\operatorname{Rad}^{S}(M)$ also has the equal images property for all $s>0$, and

(2) $M$ has constant Jordan type.

Proof. Let $K / k$ be an algebraic closure, and suppose that $\ell_{1}$ and $\ell_{2}$ are two elements of $\operatorname{Rad}(K G)$ that are not in $\operatorname{Rad}^{2}(k G)$. Then

$$
\ell_{1} \ell_{2} M=\ell_{1} \operatorname{Rad}\left(M_{K}\right)=\ell_{2} \ell_{1} M_{K}=\ell_{2} \operatorname{Rad}\left(M_{K}\right) .
$$

Because $\operatorname{Rad}\left(M_{K}\right)=\operatorname{Rad}(M)_{K}$, we have that $\operatorname{Rad}(M)$ has the equal images property by Proposition 2.6. Applying this again we get that $\operatorname{Rad}(\operatorname{Rad}(M))=\operatorname{Rad}^{2}(M)$ has the equal images property and the first statement follows by a finite induction. Moreover, we see that for $\ell_{1}, \ldots, \ell_{i} \in \operatorname{Rad}(K G) \backslash \operatorname{Rad}^{2}(K G), \ell_{i} \ldots \ell_{1}\left(M_{K}\right)=$ $\operatorname{Rad}^{i}\left(M_{K}\right)=\ell_{1}^{i}\left(M_{K}\right)$.

Now, the Jordan type of $\ell=\ell_{1}$ on $M_{K}$ is determined by the ranks of the operators $\ell^{i}, 1 \leq i<p$. Since $M$ has the equal images property, the rank of $\ell^{i}$ is the dimension of $\operatorname{Rad}^{i}\left(M_{K}\right)$. Hence $M$ has constant Jordan type by Proposition 2.1.

One pleasing aspect of the equal images property is its stability under taking quotients. The proof of the following proposition is essentially immediate, a consequence of the observation that if $M \rightarrow \bar{M}$ is a quotient map of $k G$-modules then the image of any $\alpha_{K}(t)$ on $\bar{M}$ is the quotient of the image of $\alpha_{K}(t)$ on $M$.

Proposition 2.9. Let $M$ be a $k G$-module with the equal images property and suppose that $L$ is a submodule of $M$. Then $M / L$ has the equal images property.

The above stability of the equal images property should be contrasted with the weaker property of constant Jordan type: any free $k G$-module has constant Jordan type, but most quotients do not. 


\section{3. $W$ modules and the equal images property}

In this and subsequent sections, $G$ denotes the finite group $\mathbb{Z} / p \times \mathbb{Z} / p$ and $k$ is a field of characteristic $p$. For notation, we let $k G=k\left[t_{1}, t_{2}\right] /\left(t_{1}^{p}, t_{2}^{p}\right)$, having radical generated by $x$ and $y$ which are the classes of $t_{1}$ and $t_{2}$.

As considered in [2], 2 (where the dual module is considered), the "zig-zag" modules

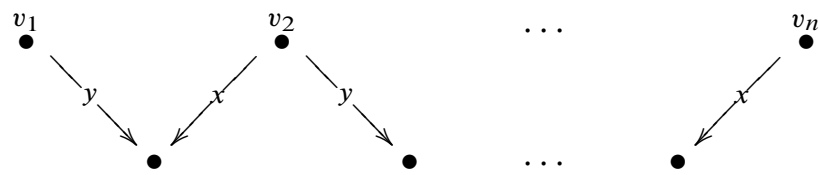

have constant Jordan type $(n-1)[2]+[1]$. The nodes in the diagram represent elements of a basis for the module. The arrows indicate the actions of $x$ and $y$ on these basis elements. We can write such a module (which we denote by $W_{n, 2}$ ) as the $k G$-module generated by elements $\left\{v_{1}, \ldots, v_{n}\right\}$ with relations generated by

$$
x v_{1}=0=y v_{n}=x^{2} v_{n} ; \quad x^{2} v_{i}=0=y v_{i}-x v_{i+1}, \quad \text { for } 1 \leq i<n-1 .
$$

For $p \geq 3$, we have the following analogue of zig-zag modules (which we denote $\left.W_{n, 3}\right)$ :

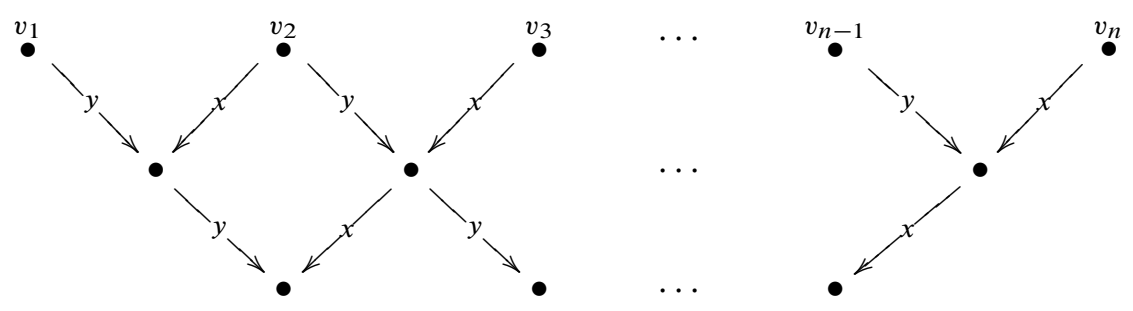

As a $k G$-module, $W_{n, 3}$ is generated by $\left\{v_{1}, \ldots, v_{n}\right\}$ with relations generated by

$$
x v_{1}=0=y v_{n}=x^{3} v_{n} ; \quad x^{3} v_{i}=0=y v_{i}-x v_{i+1}, \quad \text { for } 1 \leq i<n-1 .
$$

Clearly, the Jordan type of either $x$ or $y$ on $W_{n, 3}$ is $(n-2)[3]+[2]+[1]$. As verified more generally in Proposition $3.3, W_{n, d}$ has the equal images property so that by Proposition 2.8, it too has constant Jordan type.

We proceed to investigate these " $W$ " modules. Here is the general definition.

Definition 3.1. Let $n \geq d \geq 1$ and $d \leq p$. The $W$ module $W_{n, d}$ is the $k G$-module generated by $\left\{v_{1}, \ldots, v_{n}\right\}$ with relations generated by

$x v_{1}=0=y v_{n}=x^{d} v_{n} ; \quad x^{d} v_{i}=0=y v_{i}-x v_{i+1}, \quad$ for $1 \leq i<n-1$.

For $1 \leq n \leq d$, we set $W_{n, d}$ equal to $W_{n, n}$ as above. 
Example 3.2. As an example, we note that high powers of the radical of $k G$ are $W$ modules. Specifically, the module $\operatorname{Rad}^{p+i-1}(k G) \cong W_{p-i, p-i}$ for $i=0, \ldots, p-1$ as it has a set of generators

$$
v_{1}=x^{p-1} y^{i}, \quad v_{2}=x^{p-1} y^{i+1}, \ldots, \quad v_{p-i}=x^{i} y^{p-1}
$$

which satisfy precisely the relation of Definition 3.1, with $n=d=p-i$.

As defined, whether or not a $k G$-module $M$ is a $W$ module appears to depend upon our choice of generators $x, y$ of $\operatorname{Rad}(k G)$. But we see in Corollary 5.7, which follows, that being a $W$ module is independent of the choice of generators.

Proposition 3.3. Suppose that $n, d$ are positive integers such that $d \leq p$. Then $W_{n, d}$ has the equal images property. Thus, $W_{n, d}$ has constant Jordan type given by

$$
(n-d+1)[d]+[d-1]+\cdots+[1] .
$$

Proof. By Proposition 2.6, it suffices to verify that $\operatorname{Im}\left\{a x+b y: M_{K} \rightarrow M_{K}\right\}=$ $\operatorname{Im}\left\{x: M_{K} \rightarrow M_{K}\right\}$ for every $0 \neq(a, b) \in K^{2}$, where $M=W_{n, d}$ and $K / k$ is an algebraic closure. So assume that $k=K$ is algebraically closed. Note that $\operatorname{Rad}(M)$ is generated by the elements $y v_{1}, \ldots, y v_{n-1}$, and we know that $y v_{i}=x v_{i+1}$. So, $x W_{n, d}=y W_{n, d}=\operatorname{Rad}\left(W_{n, d}\right)$. Hence, the equal images property is a consequence of the observation that the $k$-subspace spanned by $(a x+b y) v_{1}, \ldots,(a x+b y) v_{n}$ is the same as the subspace spanned by $y v_{1}, \ldots, y v_{n-1}$.

According to Proposition 2.8, $W_{n, d}$ thus has constant Jordan type. The Jordan type $(n-d+1)[d]+[d-1]+\cdots+[1]$ is determined by inspection.

For use below, we record the following elementary properties of $W$ modules.

Proposition 3.4. Consider $W_{n, d}$ for some $n \geq d \geq 1$ and $d \leq p$. For any $i$, with $1 \leq i \leq d$,

$$
W_{n, d} / \operatorname{Rad}^{i}\left(W_{n, d}\right) \cong W_{n, i} .
$$

Furthermore, if $n_{1}, n_{2}, \ldots, n_{m}, d_{1}, d_{2}, \ldots, d_{m}$ are positive integers with $d_{i} \leq p$ for all $i$, then there exists a surjective homomorphism

$$
\zeta: W_{n, d} \rightarrow \oplus_{i=1}^{m} W_{n_{i}, d_{i}}
$$

where $n=\sum n_{i}$ and $p \geq d \geq \max \left\{d_{i}\right\}$.

Proof. The first assertion is evident from Definition 3.1.

To define a map satisfying the conditions of the second assertion we let $r_{1}=0$ and inductively let $r_{i}=r_{i-1}+n_{i-1}$ for $i=2, \ldots, m$. Note that $r_{m}+n_{m}=n$. Now let $\zeta$ be given on the generators of $W_{n, d}$ as

$$
\zeta\left(v_{r_{i}+j}\right)=v_{j}
$$


for all $1 \leq i \leq m$ and $1 \leq j \leq n_{i}$. Note here that the elements $v_{r_{i}+j}$ on the left side of the equation should be interpreted as the standard generators as in Definition 3.1 of the module $W_{n, d}$ and the element $v_{j}$ on the right side of the equation is the standard generator of $W_{n_{i}, d_{i}}$. To check that $\zeta$ is a homomorphism, we need only note that the relations among the generators of $W_{n, d}$ are also satisfied by their images under $\zeta$. In addition, $\zeta$ is surjective because every generator of the codomain is in the image of $\zeta$.

We record further natural maps of $W$ modules which we will use in the next section.

Definition 3.5. We define natural maps of $k G$-modules for any $n \geq d>1$ and $d \leq p$ :

$$
\iota_{n, d}: W_{n-1, d-1} \hookrightarrow W_{n, d}
$$

defined by identifying $W_{n-1, d-1}$ with $\operatorname{Rad}\left(W_{n, d}\right)$.

We define natural maps of $k G$-modules for any $n>d \geq 1$ and $d \leq p$ :

$$
\rho_{n, d}^{l}, \rho_{n, d}^{r}: W_{n, d} \rightarrow W_{n-1, d}
$$

where $\rho_{n, d}^{l}\left(v_{i}\right)=v_{i}, 1 \leq i<n ; \rho_{n, d}^{l}\left(v_{n}\right)=0=\rho_{n, d}^{r}\left(v_{1}\right) ; \rho_{n, d}^{r}\left(v_{i}\right)=v_{i-1}$, $1<i \leq n$.

We conclude this section with a useful statement concerning the generation of the indecomposable $W$ module $M=W_{n, d}$. Let $v_{1}, \ldots, v_{n}$ be generators for $M$ satisfying the relations of Definition 3.1. Let $(a, b)$ be any pair of elements of $k$, which are not both zero. Then the element

$$
v_{a, b}=a^{n-1} v_{1}-a^{n-2} b v_{2}+a^{n-3} b^{2} v_{3}+\cdots+(-1)^{n-1} b^{n-1} v_{n}
$$

has the property that

$$
(a x+b y) v_{a, b}=0 .
$$

This is a straightforward verification using the relations that $x v_{1}=0, y v_{1}=x v_{2}$, and so forth.

Proposition 3.6. Let $M=W_{n, d}$ for some $n \geq d \geq 1$. Suppose that the pairs $\left(a_{1}, b_{1}\right), \ldots,\left(a_{n}, b_{n}\right)$ in $k^{2}$ have the property that the classes $\left\langle a_{1}, b_{1}\right\rangle, \ldots,\left\langle a_{n}, b_{n}\right\rangle$ are mutually distinct elements of $\mathbb{P}^{1}(k)$. Then

$$
M=\sum_{i=1}^{n} \operatorname{Ker}\left\{\left(a_{i} x+b_{i} y\right): M \rightarrow M\right\} .
$$


Proof. For notational convenience, let $\mathcal{K}$ denote $\sum_{i=1}^{n} \operatorname{Ker}\left\{\left(a_{i} x+b_{i} y\right): M \rightarrow M\right\}$. To prove the proposition, it suffices to show that each $v_{i}$ lies in $\mathcal{K}$. To prove this, we show that the $k$-subspace $V$ spanned by $v_{1}, \ldots, v_{n}$ coincides with the $k$-subspace $W \subseteq \mathcal{K}$ spanned by $v_{a_{1}, b_{1}}, \ldots, v_{a_{n}, b_{n}}$. Clearly, $W \subseteq V$, since each $v_{a_{i}, b_{i}}$ is in $V$. Consequently, if we show that $W$ has dimension $n$, then $W=V, V \subseteq \mathcal{K}$ and $M=\mathcal{K}$.

To prove that $W$ has dimension $n$, it suffices to show that the elements $v_{a_{i}, b_{i}}$, for $i=1, \ldots, n$, are linearly independent. This is equivalent to the non-vanishing of the determinant

$$
\operatorname{Det}\left(\begin{array}{llll}
a_{1}^{n-1} & a_{1}^{n-2} b_{1} & \ldots & b_{1}^{n-1} \\
a_{2}^{n-1} & a_{2}^{n-2} b_{2} & \ldots & b_{2}^{n-1} \\
& & & \\
a_{n}^{n-1} & a_{n}^{n-2} b_{n} & \ldots & b_{n}^{n-1}
\end{array}\right) .
$$

The above is a Vandermonde determinant (formally factor $a_{i}^{n-1}$ out of the $i^{\text {th }}$ row to put it in the form given in [10]). Formally, it is a polynomial in the variables $a_{1}, \ldots, a_{n}, b_{1}, \ldots, b_{n}$ and has value $\prod_{i<j}\left(a_{j} b_{i}-a_{i} b_{j}\right)$. Consequently, because the elements $\left\langle a_{1}, b_{1}\right\rangle, \ldots,\left\langle a_{n}, b_{n}\right\rangle$ are distinct, we have the determinant is not zero. Hence the dimension of $W$ is $n$ and the proposition is proved.

\section{The Quillen exact category of $W$ modules}

We briefly recall for our context of $W$ modules the Quillen exact category structure considered in [1]. We shall be interested in the case $G=\mathbb{Z} / p \times \mathbb{Z} / p$, but the following definitions apply to any finite group scheme.

Let

$$
\varepsilon: \quad 0 \longrightarrow M_{1} \longrightarrow M_{2} \longrightarrow M_{3} \longrightarrow 0
$$

be any short exact sequence of finite dimensional $k G$-modules. Then this sequence is said to be locally split if for any $\pi$-point $\alpha_{K}: K[t] /\left(t^{p}\right) \rightarrow K G$ the pull-back of $\mathscr{E}$ via $\alpha_{K}, 0 \rightarrow \alpha_{K}\left(M_{1, K}\right) \rightarrow \alpha_{K}\left(M_{2, K}\right) \rightarrow \alpha_{K}\left(M_{3, K}\right) \rightarrow 0$ is a split exact sequence. An admissible monomorphism, denoted $M_{1} \hookrightarrow M_{2}$, is an injective map of $k G$-modules which fits into a locally split short exact sequence; an admissible epimorphism, denoted $M_{2} \rightarrow M_{3}$, is a surjective map of $k G$-modules which fits into a locally split short exact sequence.

As in [1], it is straightforward to verify that the following determines an exact subcategory of the Quillen exact category of finite dimensional $k G$-modules with locally split short exact sequences.

Definition 4.1. We denote by $\mathcal{C}$ the Quillen exact category of modules of constant Jordan type as considered in [1]. We denote by $\ell W$ the additive full subcategory 
generated by $k G$-modules of the form $W_{n, d}$. Then $\ell W \subset \ell$ inherits the structure of a Quillen exact category.

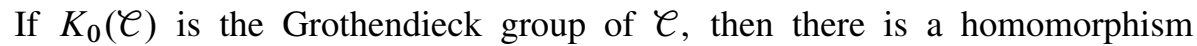
JType: $K_{0}(\mathcal{C}) \rightarrow \mathbb{Z}^{p}$, which takes the class of $M$ to its Jordan type $\operatorname{JType}(M)$ at any $\pi$-point.

Theorem 4.2. Let $\iota: € W \rightarrow €$ be the inclusion of Quillen exact categories and let $\iota_{*}: K_{0}(\mathcal{C W}) \rightarrow K_{0}(\mathcal{C})$ denote the induced map on Grothendieck groups. Then the composition

$$
\text { JType } \circ \iota_{*}: K_{0}(\mathcal{C W}) \rightarrow \mathbb{Z}^{p}
$$

is an isomorphism. Thus, $\iota_{*}$ gives a left splitting of the Jordan type homomorphism

$$
\text { JType: } K_{0}(\mathcal{C}) \rightarrow \mathbb{Z}^{p} \text {. }
$$

The group $K_{0}(\mathcal{C W})$ is minimally generated by the set of classes $\left\{\left[W_{n, n}\right] \mid 1 \leq n \leq p\right\}$.

Proof. Surjectivity of JType $\circ \iota_{*}$ is clear, for the images under JType $\circ \iota_{*}$ of the classes of the modules

$$
W_{p, p}, \quad W_{p-1, p} \equiv W_{p-1, p-1}, \ldots, W_{2, p}, W_{1, p}=k
$$

generate $\mathbb{Z}^{p}$. Indeed, by (2) we see that

$$
\operatorname{JType}\left(W_{n, p}\right)=[n]+[n-1]+\cdots+[1], \quad 1 \leq n \leq p .
$$

Thus, $\left\{W_{n, p} \mid 1 \leq n \leq p\right\}$ is also a linearly independent subset of $K_{0}(\mathcal{C W})$.

To prove injectivity, we first observe the existence of a locally split exact sequence

$$
0 \longrightarrow W_{n, d} \stackrel{\sigma}{\longrightarrow} W_{n-1, d} \oplus W_{n-1, d} \stackrel{\tau}{\longrightarrow} W_{n-2, d} \longrightarrow 0
$$

whenever $n-1 \geq d \geq 1$ and $d \leq p$. Here we have $\sigma=\left(\rho_{n, d}^{l}, \rho_{n, d}^{r}\right)$ and $\tau=$ $\left(\rho_{n-1, d}^{r},-\rho_{n-1, d}^{l}\right)$, with $\rho_{n, d}^{l}, \rho_{n, d}^{r}$ as in (5). Note that each of these sequences is locally split because the middle term has precisely the same (constant) Jordan type as the direct sum of the two ends. The sequences in (10) provide the relations

$$
\left[W_{n, d}\right]=2\left[W_{n-1, d}\right]-\left[W_{n-2, d}\right]=0 ; \quad n-1 \geq d \geq 1, d \leq p .
$$

These relations easily enable us to write any $W_{n, d}$ as an integral linear combination of $W_{m, p} \equiv W_{m, m}$ with $m \leq d \leq p$. In particular, we conclude that $K_{0}(\mathcal{C W})$ is generated by $\left\{W_{n, p} \mid 1 \leq n \leq p\right\}$. 


\section{The ubiquity of $W$ modules}

Proposition 2.8 tells us that modules with the equal images property are modules of constant Jordan type. In Theorem 5.4, we verify that every module with the equal image property is a quotient of some $W$ module.

Using the classification by A. Heller and I. Reiner [11] of $k G$-modules with radical square equal to 0 , we identify those modules which also have the equal images property.

Proposition 5.1. Assume that $M$ is a $k G$-module having the equal images property and satisfying $\operatorname{Rad}^{2}(M)=\{0\}$. Then $M$ is isomorphic to a direct sum of $W$ modules. More precisely, there exist integers $s, t$ and $n_{1}, \ldots, n_{t}$ such that

$$
M \cong W_{n_{1}, 2} \oplus W_{n_{1}, 2} \oplus \cdots \oplus W_{n_{t}, 2} \oplus k^{s}
$$

Proof. The indecomposable $k G$-modules $M$ with $\operatorname{Rad}^{2}(M)=\{0\}$ have been completely classified in [11]. As pointed out in [1], using this classification it is an easy exercise to verify that if an indecomposable $k G$-module $M$ with $\operatorname{Rad}^{2}(M)=\{0\}$ has the equal images property then $M$ is isomorphic to some $W_{n, 2}$ or $k$.

From the above, we get some information on the Jordan types of modules with the equal images property.

Proposition 5.2. Suppose that $M$ has the equal images property and that the (constant) Jordan type of $M$ is $a_{p}[p]+a_{p-1}[p-1]+\cdots+a_{1}[1]$. Then there exists $a$ non-negative integer $s$ such that $a_{i}=0$ for $i>s$ and $a_{i} \neq 0$ for $i \leq s$.

Proof. Let $n_{i}=\operatorname{Dim}\left(\operatorname{Rad}^{i-1}(M)\right)-\operatorname{Dim}\left(\operatorname{Rad}^{i}(M)\right)$. Recall from Section 2, that $x^{i} M=\operatorname{Rad}^{i}(M)$ because of the equal images property. Hence, $n_{i}$ is the number of Jordan blocks of the action of $x$ on $M$ that have size at least $i$. Let $s$ be the greatest integer such that $n_{s} \neq 0$ or equivalently, such that $\operatorname{Rad}^{s-1}(M) \neq\{0\}$. Clearly, $a_{i}=0$ for $i>s$, since the operation of $x$ on $M$ has no Jordan blocks of size larger than $s$. Moreover, $a_{s}=n_{s}$ and for $i<s$, we have that $a_{i}=n_{i}-n_{i-1}$.

Now by Propositions 2.8 and 2.9, the modules $N_{i}=\operatorname{Rad}^{i-1}(M) / \operatorname{Rad}^{i+1}(M)$ have the equal images property for all $i$. Moreover, $\operatorname{Rad}^{2}\left(N_{i}\right)=\{0\}$. Hence by Proposition 5.1, each $N_{i}$ is a sum of $\mathrm{W}$ modules of the form $W_{m, 2}$. It follows that

$$
n_{i}=\operatorname{Dim}\left(N_{i} / \operatorname{Rad}\left(N_{i}\right)\right)>\operatorname{Dim}\left(\operatorname{Rad}\left(N_{i}\right)\right)=n_{i+1}
$$

for all $i=1, \ldots, s$. Consequently, $a_{i} \neq 0$ for $1 \leq i \leq s$.

Our next proposition is the key to establishing that modules with the equal images property are quotients of direct sums of $W$ modules. 
Proposition 5.3. Suppose that $M$ has the equal images property and that $m$ is an element of $M$. For $n$ sufficiently large, there exists a map $\psi: W_{n, p} \rightarrow M$ such that for some $s, \psi\left(v_{s}\right)=m$ where $v_{s} \in W_{n, p}$ is a generator as in the notation of Definition 3.1.

Proof. We first verify that for some $t$, there exist elements $m_{0}, m_{1} \ldots, m_{t}$ such that

$$
m_{0}=m ; \quad y m_{i}=x m_{i+1} \quad \text { for } i=0, \ldots, t-1 ; \quad y m_{t}=0 .
$$

We proceed by induction on $r$ where $r$ is the least integer such that $\operatorname{Rad}^{r}(M)=\{0\}$. In the case that $r=1, M$ is a sum of trivial modules, so that we may assume that $t=1$. If $r=2$, then we use Proposition 5.1 and the explicit structure of $W_{n, 2}$ to obtain $m_{0}, m_{1} \ldots, m_{t}$ satisfying (13).

Proceeding inductively, we may assume that $m \notin \operatorname{Rad}(M)$. For if $m \in \operatorname{Rad}(M)$, we appeal to our induction hypothesis to find elements $m_{0}, m_{1} \ldots, m_{t}$ in $\operatorname{Rad}(M)$ satisfying (13) since $\operatorname{Rad}(M)$ also has the equal images property. By Proposition 5.1, there exist integers $q$ and $n_{1}, \ldots, n_{q}$, (some of which may be equal to 1 ) such that $M / \operatorname{Rad}^{2}(M) \cong W_{n_{1}, 2} \oplus \cdots \oplus W_{n_{q}, 2}$. Consequently, if $n$ is the maximum of the integers $n_{1}, \ldots, n_{q}$, then $n \geq 2$ and we can find elements $m_{0}, \ldots, m_{n}$ such that $m_{0}=$ $m$ and for all $i=0, \ldots, n-1, y m_{i}=x m_{i+1}$ and also that $y m_{n} \in \operatorname{Rad}^{2}(M)$. Then by induction we can find $m_{n+1}, \ldots m_{t}$ in $\operatorname{Rad}(M)$ for some $t$ so that $y m_{i}=x m_{i+1}$ for $i=n, \ldots, t-1$ and that $y m_{t}=0$.

Interchanging the roles of $x$ and $y$, we conclude that there exist an integer $s \geq 1$ and elements $m_{0}, \ldots, m_{-s}$ such that

$$
m=m_{0} ; \quad y m_{i-1}=x m_{i} \text { for } i=0, \ldots,-s+1 ; \quad x m_{-s}=0 .
$$

Hence, we obtain a well defined $k G$-homomorphism

$$
\psi: W_{s+t+1, p} \rightarrow M ; \quad \psi\left(v_{i}\right)=m_{-s-1+i} .
$$

Theorem 5.4. Suppose that $M$ is a $k G$-module. Then $M$ has the equal images property if and only if there exist a positive integer $n$ and a surjective homomorphism

$$
\psi: W_{n, d} \rightarrow M
$$

where d satisfies $\operatorname{Rad}^{d}(M)=0$.

Proof. If such a map $\psi$ exists, then $M$ has the equal images property by Lemma 2.9. Hence, we assume that $M$ has the equal images property. Our objective is to construct the map $\psi$.

Let $m_{1}, \ldots, m_{n}$ be a collection of elements such that the cosets $m_{i}+\operatorname{Rad}(M)$ for $i=1, \ldots, n$ form a basis for $M / \operatorname{Rad}(M)$. By Proposition 5.3, for each $i$ there 
is an integer $n_{i}$ and a homomorphism $\psi_{i}: W_{2 n_{i}, p} \rightarrow M$ such that $\psi_{i}\left(v_{n_{i}}\right)=m_{i}$. Thus by Nakayama's Lemma we have that the sum of the maps

$$
\Psi: \bigoplus_{i=1}^{n} W_{2 n_{i}, p} \rightarrow M
$$

given by $\Psi\left(w_{1}, \ldots, w_{n}\right)=\sum \psi_{i}\left(w_{i}\right)$ is surjective. Moreover, each summand of this map clearly factors through $W_{2 n_{i}, p} / \operatorname{Rad}^{d}\left(W_{2 n_{i}, p}\right)$. The proof of the theorem is thus completed by an appeal to Proposition 3.4.

The following corollary of Theorem 5.4 will be key to our discussion in Section 7 of generic kernels.

Corollary 5.5. Suppose that $k$ is an infinite field and that $S \subset \mathbb{P}^{1}(k)$ is infinite. Let $M$ be any $k G$-module having the equal images property. Then

$$
M=\sum_{\langle a, b\rangle \in S} \operatorname{Ker}\{a x+b y: M \rightarrow M\} .
$$

Proof. By Theorem 5.4, there exist an integer $n$ and a surjective homomorphism $\psi: W_{n, p} \rightarrow M$. To prove the theorem we need only note that for any pair $(a, b) \neq$ $(0,0)$ in $k^{2}$

$$
\psi\left(\operatorname{Ker}\left\{a x+b y: W_{n, p} \rightarrow W_{n, p}\right\}\right) \subseteq \operatorname{Ker}\{a x+b y: M \rightarrow M\} .
$$

Then by Proposition 3.6, we have that

$$
\begin{aligned}
M=\psi\left(W_{n, p}\right) & =\sum_{(a, b) \in S} \psi\left(\left\{m \in W_{n, p} \mid(a x+b y) m=0\right\}\right) \\
& \subseteq \sum_{(a, b) \in S}\{m \in M \mid(a x+b y) m=0\},
\end{aligned}
$$

thus proving the corollary.

If $M / \operatorname{Rad}^{2}(M)$ is indecomposable and if $M$ has the equal images property, then we give in Proposition 5.6 a "much more efficient" surjection from a $W$ module onto $M$ than that described in the proof of Theorem 5.4..

Proposition 5.6. Assume that $M$ is a $k G$-module having the equal images property. If $M / \operatorname{Rad}^{2}(M)$ is indecomposable, then $M$ is a quotient of the $W$ module $W_{n, p}$ for $n=\operatorname{Dim}(M / \operatorname{Rad}(M))$.

Proof. Because $M / \operatorname{Rad}^{2}(M)$ is indecomposable, Proposition 5.1 implies the existence of generators $a_{1}, \ldots, a_{n}$ with the property that

$$
y a_{n} \equiv 0, \quad x a_{1} \equiv 0, \quad \text { and } \quad y a_{i} \equiv x a_{i+1}
$$


modulo $\operatorname{Rad}^{2}(M)$ where $i=1, \ldots, n-1$. Moreover, the equal images property for $M$ implies that

$$
\operatorname{Rad}^{2}(M)=x^{2} M=x y M=y^{2} M .
$$

We proceed to modify the set $\left\{a_{1}, \ldots, a_{n}\right\}$ of generators in order to obtain a new set of generators satisfying the conditions on $\left\{v_{1}, \ldots, v_{n}\right\}$ given in Definition 3.1. Our first step involves observing that $x a_{1}$ is in $\operatorname{Rad}^{2}(M)$; thus, $x a_{1}=x^{2} w$ for some $w$. Replace $a_{1}$ by $a_{1}^{\prime}=a_{1}-x w$, so that $x a_{1}=0$. Since $y a_{1}^{\prime}-x a_{2}=x^{2} w^{\prime}$ for some $w^{\prime}$ in $\operatorname{Rad}^{2}(M)$, we may replace $a_{2}$ by $a_{2}^{\prime}=a_{2}+x w^{\prime}$, so that $y a_{1}^{\prime}=x a_{2}^{\prime}$. Continuing, we obtain $\left\{a_{1}^{\prime}, \ldots, a_{n}^{\prime}\right\}$ such that

$$
x a_{1}^{\prime}=0, \quad \text { and } y a_{i}^{\prime}=x a_{i+1}^{\prime}
$$

for $i=1, \ldots, n-1$.

There remains to modify this new generating set $\left\{a_{1}^{\prime}, \ldots, a_{n}^{\prime}\right\}$ to obtain a generating set which satisfies the above relations and further satisfies the relation that $y a_{n}^{\prime}=0$. Since $y a_{n}^{\prime}$ is in $\operatorname{Rad}^{2}(M)$, we may choose $\ell_{1}, \ldots, \ell_{n-2} \in k$ such that

$$
y a_{n}^{\prime} \equiv y^{2}\left(\ell_{1} a_{1}^{\prime}+\cdots+\ell_{n-2} a_{n-2}^{\prime}\right) \text { modulo } \operatorname{Rad}^{3}(M) .
$$

Observe that we do not need to add a term of the form $x^{2} \ell_{n-1} a_{n-1}^{\prime}$, because $y a_{n-1}^{\prime}=$ $x a_{n}^{\prime}$ so that $y^{2} a_{n-1}^{\prime}=x y a_{n}^{\prime}$ lies in $\operatorname{Rad}^{3}(M)$.

Set

$$
b_{1}=a_{1}^{\prime}, \quad b_{2}=a_{2}^{\prime}, \quad b_{3}=a_{3}^{\prime}-x \ell_{n-2} a_{1}^{\prime}, \ldots, \quad b_{i}=a_{i}^{\prime}-x \sum_{j=1}^{i-2} \ell_{n-i+j} a_{j}^{\prime}, \ldots
$$

A straightforward calculation confirms that

$$
x b_{1} \equiv 0, \quad y b_{n} \equiv 0, \quad \text { and } \quad y b_{i} \equiv x b_{i+1} \quad \operatorname{modulo} \operatorname{Rad}^{3}(M) .
$$

We continue this process, inductively obtaining the corresponding set of relations modulo the submodule $\operatorname{Rad}^{j}(M)$, stopping at $j=p$, since by Proposition 5.3, $\operatorname{Rad}^{p}(M)=\{0\}$.

Corollary 5.7. Let $x^{\prime}, y^{\prime} \in k G$ be a choice of generators of $\operatorname{Rad}(k G)$, let $n \geq d \geq 1$ with $d \leq p$, and let $M$ be a $k G$-module generated by $\left\{w_{1}, \ldots, w_{n}\right\}$ subject to the relations generated by

$$
x^{\prime} w_{1}=0=y^{\prime} w_{n} ; \quad\left(x^{\prime}\right)^{d} w_{i}=0 ; \quad y^{\prime} w_{i}=x^{\prime} w_{i+1}, \quad 1 \leq i<d .
$$

Then $M$ is isomorphic to $W_{n, d}$.

Proof. As argued in the proof of Proposition 3.3, $M$ has the equal images property. Since $M$ clearly satisfies the condition that $M / \operatorname{Rad}^{2}(M)$ is indecomposable, Proposition 5.6 implies that $M$ is isomorphic to $W_{n, d}$. 
The following proposition verifies that there are a great many $k G$-modules of constant Jordan type. This is because any quotient of a $\mathrm{W}$ module must have the equal images property and thus also constant Jordan type.

Proposition 5.8. Suppose that $M=W_{n, d}$ for some $n>4$ and $3 \leq d \leq p$. Suppose that $L$ and $N$ are subspaces of $\operatorname{Soc}(M)$ (the socle of $M$ ) which in this case is equal to $\operatorname{Rad}^{d-1}(M)$. There exists an isomorphism $\varphi: M / L \stackrel{\sim}{\longrightarrow} M / N$ of $k G$-modules only if $L=N$.

Proof. Recall that $\operatorname{Soc}(M)$ is the sum of the irreducible submodules of $V$. Hence, $\operatorname{Soc}(M)$ is a trivial $k G$-module and $L, N$ are $k G$-submodules of $M$. If $M$ is generated by $v_{1}, \ldots, v_{n}$ as in Definition 3.1, then $\operatorname{Soc}(M)$ is the subspace of $M$ generated by $y^{d-1} v_{1}, \ldots, y^{d-1} v_{n-d+1}$ and both $x$ and $y$ annihilate the subspaces $L$ and $N$.

We assume that $v_{1}, \ldots, v_{n}$ are the generators of $M$ with precisely the relations as in Definition 3.1. Suppose that $\ell=a x-b y$ for $(a, b) \neq(0,0)$ in $k^{2}$. Multiplication by $\ell$ induces a surjective homomorphism

$$
\ell: M / \operatorname{Rad}(M) \rightarrow \operatorname{Rad}(M) / \operatorname{Rad}^{2}(M)
$$

whose kernel has dimension one and is generated by the class of the element $m_{\ell}=$ $a^{n-1} v_{1}+a^{n-2} b v_{2}+\cdots+b^{n-1} v_{n}$. In particular, we have that $\ell \cdot m_{\ell}=0$. This is a straightforward verification using the generators and relations.

We claim that if $m \in M / L, m \notin \operatorname{Rad}(M / L)$, with $\ell m=0$, then $m \equiv \mathrm{cm}_{\ell}$ modulo $\operatorname{Rad}(M / L)$ for some non-zero $c \in k$. The reason is that $L \subseteq \operatorname{Rad}^{d-1}(M)$ for $d \geq 3$, and hence

$$
M / \operatorname{Rad}(M) \simeq(M / L) / \operatorname{Rad}(M / L)
$$

and

$$
\operatorname{Rad}(M) / \operatorname{Rad}^{2}(M) \simeq \operatorname{Rad}(M / L) / \operatorname{Rad}^{2}(M / L)
$$

where the isomorphisms are induced by the quotient map $M \rightarrow M / L$. The same claim holds for $M / N$.

Assume that $\varphi: M / L \rightarrow M / N$ is an isomorphism. We define $\theta=q_{2}^{-1} \varphi^{\prime} q_{1}$, so that $\theta$ fits in the commutative diagram

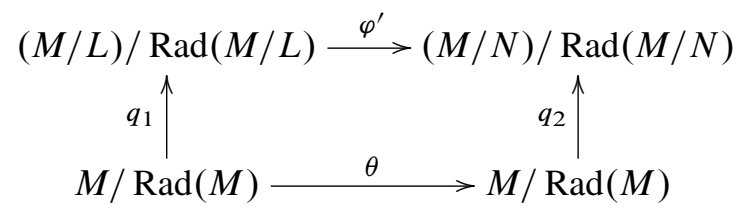

where $\varphi^{\prime}$ is the map induced by $\varphi$, and the two vertical arrows are isomorphisms induced by the natural quotient maps. The commutativity of (15) implies

$$
\varphi\left(m_{\ell}+L\right)=c_{\ell} m_{\ell}+N \text { modulo } \operatorname{Rad}(M / N),
$$


for some constant element $c_{\ell}$. In particular, we get that

$$
\theta\left(m_{\ell}+\operatorname{Rad}(M)\right)=c_{\ell} m_{\ell}+\operatorname{Rad}(M) .
$$

In other words, the class of $m_{\ell}$ is an eigenvector for the map of $\theta$ with eigenvalue $c_{\ell}$.

Now because the field $k$ is infinite, we may choose $n$ distinct scalars $b_{1}, \ldots, b_{n}$ in $k$ and form the elements $\ell_{i}=x+b_{i} y$. Then we have $n$ elements

$$
m_{\ell_{i}}=m_{i}=v_{1}+b_{i} v_{2}+b_{i}^{2} v_{3}+\cdots+b_{i}^{n-1} v_{n}
$$

Observe that the matrix of coefficients of this collection of elements has the form

$$
\left(\begin{array}{llll}
1 & b_{1} & \ldots & b_{1}^{n-1} \\
1 & b_{2} & \ldots & b_{2}^{n-1} \\
& & \ldots & \\
1 & b_{n} & \ldots & b_{n}^{n-1}
\end{array}\right)
$$

which is a Vandermonde matrix. Its determinant is $\prod_{i \leq j}\left(b_{i}-b_{j}\right) \neq 0$ and we conclude that the set of classes of the elements $m_{1}, \ldots, m_{n}$ is a basis for $M / \operatorname{Rad}(M)$. As a consequence, the matrix of $\theta$ with respect to this basis is a diagonal matrix. We now show that it is a scalar matrix.

Suppose that $\ell=x+b y$, where $b \in k$ is not equal to any of $b_{1}, b_{2}, \ldots, b_{n}$. Then there exist $a_{1}, \ldots, a_{n} \in k$ such that

$$
m_{\ell}=v_{1}+b v_{2}+\cdots+b^{n-1} v_{n}=a_{1} m_{1}+\cdots+a_{n} m_{n} .
$$

Note that $a_{i} \neq 0$ for all $i$. This is because, if $a_{i}=0$, then substituting the row $\left(\begin{array}{llll}1 & b & \ldots & b^{n-1}\end{array}\right)$ for the $i^{\text {th }}$ row of the above matrix, we get a Vandermonde matrix with zero determinant which is not possible.

Applying $\theta$ to the above expression, we get that $c_{\ell}=c_{\ell_{i}}$ for every $i$ since the $m_{i}$ 's are linearly independent. Hence, we have that for any $v$ in $M / \operatorname{Rad}(M), \theta(v)=c v$ where $c=c_{\ell}$ for any (and every) $\ell$. In particular, we have that $\varphi(m+L) \equiv c m+N$ $\bmod \operatorname{Rad}(M / N)$ for any $m \in M$.

At the level of $\operatorname{Rad}^{d-1}(M / L)$, we have that

$$
\varphi\left(y^{d-1} v_{i}+L\right)=c y^{d-1} v_{i}+N \text { for } i=1, \ldots, n-d+1,
$$

since $\operatorname{Rad}^{d}(M)=0$. Hence, for any element $m \in L, m$ can be expressed as a linear combination of the elements $y^{d-1} v_{1}, \ldots, y^{d-1} v_{n-d+1}$. It follows that $0=$ $\varphi(m+L)=c m+N$, and $m \in N$. Therefore $L=N$.

We end this section with the following question on the possibility of classifying modules of constant Jordan type. We remind the reader that if a module category has wild representation type, then it is generally considered that its objects can not 
be classified in any reasonable sense. Specifically, if the module category is wild then there is an embedding of the category of finite dimensional $k\{x, y\}$-modules into the module category (cf. [4]); here, $k\{x, y\}$ is the associative $k$-algebra freely generated by two non-commuting elements. Consequently, a classification of objects in the module category would imply the existence of a canonical form for pairs of non-commuting $n \times n$ matrices for all $n$. Such a form is unlikely to exist.

Question 5.9. Does the full subcategory of the category of finitely generated $k G$ modules consisting of all modules of constant Jordan type have wild representation type?

\section{Associated bundles on $\mathbb{P}^{1}$}

In [6], a construction is presented which associates to a finite dimensional $k G$-module $M$ of constant Jordan type a family of algebraic vector bundles

$$
\left\{\operatorname{Ker}\left\{\widetilde{\Theta}_{G}^{j}, \mathcal{O}_{\operatorname{Proj} k[V(G)]} \otimes M\right\} \mid 1 \leq j<p\right\}
$$

on the projectivization of the affine $k$-scheme $V(G)$ of 1-parameter subgroups of $G$. Since the group algebra of $\mathbb{Z} / p \times \mathbb{Z} / p$ is isomorphic to the group algebra of the infinitesimal group scheme $\mathbb{G}_{a(1)} \times \mathbb{G}_{a(1)}$, we may view $W$ modules for $\mathbb{Z} / p \times \mathbb{Z} / p$ as modules (of constant Jordan type) for $\mathbb{G}_{a(1)} \times \mathbb{G}_{a(1)}$. For $G=\mathbb{G}_{a(1)} \times \mathbb{G}_{a(1)}$, $V(G)$ is simply the 2 -dimensional affine space $\mathbb{A}^{2}$ over $k$ so that $\operatorname{Proj} k[V(G)] \simeq \mathbb{P}^{1}$, the projective line.

In this section, we set $G=\mathbb{G}_{a(1)} \times \mathbb{G}_{a(1)}$ and set $\mathcal{O}=\mathcal{O}_{\operatorname{Proj} k[V(G)]}=\mathcal{O}_{\mathbb{P}}$. In the proposition below, we identify the bundles

$$
\left\{\operatorname{Ker}\left\{\widetilde{\Theta}_{G}^{j}, \mathcal{O} \otimes W_{n, d}\right\} \mid j=1,2\right\} .
$$

The general construction of $\operatorname{Ker}\left\{\widetilde{\Theta}_{G}^{j}, \mathcal{O}_{\operatorname{Proj} k[V(G)]} \otimes M\right\}$ specialized to $G=$ $\mathbb{G}_{a(1)} \times \mathbb{G}_{a(1)}$ can be described concretely as follows. Write $k G=k[x, y] /\left(x^{p}, y^{p}\right)$. We associate to any finite dimensional $k G$-module $M$ the following endomorphism $k[s, t] \otimes M \rightarrow k[s, t] \otimes M, \quad f(s, t) \otimes m \mapsto s f(s, t) \otimes(x \cdot m)+t f(s, t) \otimes(y \cdot m)$.

We view this endomorphism as the map on the $k[s, t] \otimes k G$-module $k[s, t] \otimes M$ given by multiplication by

$$
\Theta_{G}=s \otimes x+t \otimes y \in k[s, t] \otimes k G
$$

Because $\Theta_{G}$ is homogeneous of weight 1 (with respect to the grading on $k[s, t]$ assigning both $s, t$ weight 1 ), we obtain a projectivization of our endomorphism

$$
\widetilde{\Theta}_{G}: \mathcal{O} \otimes M \rightarrow \mathcal{O}(1) \otimes M,
$$

a map of coherent $\mathcal{O}$-modules on the scheme $\mathbb{P}^{1}$. 
Here, and below, we extend our notational shorthand of $\mathcal{O}$ for $\mathcal{O}_{\mathbb{P} 1}$ by letting $\mathcal{O}(n)$ denote $\mathcal{O}_{\mathbb{P}^{1}}(n)$.

Proposition 6.1 (cf. [6], 5.1). As above, let $G=\mathbb{G}_{a(1)} \times \mathbb{G}_{a(1)}$, and let $M$ be a finite dimensional $k G$-module. Let $\alpha_{a, b}: K[u] /\left(u^{p}\right) \rightarrow K G$ be the (flat) map of $K$-algebras sending $u$ to $a x+b y$ for any $(a, b) \in \mathbb{A}(K)^{2}-\{0\}$, where $K / k$ is some field extension. Then the action of $\alpha_{a, b}(u)$ on $M_{K}=K \otimes M$ is given by restricting along $k[s, t] \rightarrow K$ via $s \mapsto a, t \mapsto b$ the action of multiplication by $\Theta_{G}$ on $k[s, t] \otimes M$.

In particular, if for a given $j, 1 \leq j<p$, the rank of $\alpha_{a, b}^{j}: M \rightarrow M$ is independent of $(a, b) \neq 0$ (i.e., if $M$ has constant $j$-type in the terminology of [6]), then the kernel of $\widetilde{\Theta}_{G}^{j}$ on $\mathcal{O} \otimes M$ is an algebraic vector bundle on $\mathbb{P}^{1}$, sub-bundle of the trivial bundle $\mathcal{O} \otimes M$ :

$$
\operatorname{Ker}\left\{\widetilde{\Theta}_{G}^{j}, \mathcal{O} \otimes M\right\} \subset \mathcal{O} \otimes M .
$$

Moreover, the image of $\widetilde{\Theta}_{G}^{j}$ on $\mathcal{O} \otimes M$ is an algebraic vector bundle on $\mathbb{P}^{1}(k)$, a sub-bundle of $\mathcal{O}(j) \otimes M$ :

$$
\operatorname{Im}\left\{\widetilde{\Theta}_{G}^{j}, \mathcal{O} \otimes M\right\} \subset \mathcal{O}(j) \otimes M .
$$

Remark 6.2. The infinitesimal group scheme $\mathbb{G}_{a(2)}$ also has group algebra isomorphic to the group algebra of $\mathbb{Z} / p \times \mathbb{Z} / p$. The affine scheme $V\left(\mathbb{G}_{a(2)}\right)$ of 1-parameter subgroups can be identified with the spectrum of the graded polynomial algebra $k\left[s_{0}, s_{1}\right]$ with $\operatorname{deg}\left(s_{0}\right)=1, \operatorname{deg}\left(s_{1}\right)=p$. The associated projective scheme is a weighted $\mathbb{P}^{1}$ isomorphic to $\mathbb{P}^{1}$ itself. This contrasts sharply with the rank 3 case: $\operatorname{Proj} k\left[V\left(\mathbb{G}_{a(1)} \times \mathbb{G}_{a(1)} \times \mathbb{G}_{a(1)}\right)\right] \simeq \mathbb{P}^{2}$ whereas Proj $k\left[V\left(\mathbb{G}_{a(3)}\right)\right]$ is the weighted projective space $\mathbb{P}\left(1, p, p^{2}\right)$ which is a singular variety.

The reader can readily check that the bundles on $\mathbb{P}^{1} \simeq \operatorname{Proj} k\left[V\left(\mathbb{G}_{a(1)} \times \mathbb{G}_{a(1)}\right)\right]$ associated to a $\mathbb{Z} / p \times Z / p$-module $M$ of constant Jordan type arising when we use the infinitesimal group scheme $\mathbb{G}_{a(1)} \times \mathbb{G}_{a(1)}$ are isomorphic to those arising when we use the infinitesimal group scheme $\mathbb{G}_{a(2)}$, once we identify Proj $k\left[V\left(\mathbb{G}_{a(1)} \times \mathbb{G}_{a(1)}\right)\right]$ with $\operatorname{Proj} k\left[V\left(\mathbb{G}_{a(2)}\right)\right]$.

The following proposition is essentially given by Proposition 6.13 in [6].

Proposition 6.3. Let us use the isomorphism of the group algebra of $\mathbb{Z} / p \times \mathbb{Z} / p$ with the group algebra of $k G, G=\mathbb{G}_{a(1)} \times \mathbb{G}_{a(1)}$, in order that we may view $W_{n, 2}$ as a $k G$-module. Then

$$
\operatorname{Ker}\left\{\widetilde{\Theta}_{G}, \mathcal{O} \otimes W_{n, 2}\right\} \simeq \mathcal{O}^{\oplus n-1} \oplus \mathcal{O}(-n+1) .
$$


Proof. Observe that

$$
\operatorname{Im}\left\{\widetilde{\Theta}_{G}, \mathcal{O} \otimes W_{n, 2}\right\} \otimes \mathcal{O}(-1) \subset \mathcal{O} \otimes W_{n, 2}
$$

is a free module of rank $n-1$ on $\mathbb{P}^{1}$. Hence, we may identify this image with $\mathcal{O} \otimes$ $\operatorname{Rad}\left(W_{n, 2}\right)$. As shown in Proposition 6.13 of [6], the quotient of $\operatorname{Ker}\left\{\widetilde{\Theta}_{G}, \mathcal{O} \otimes W_{n, 2}\right\}$ modulo $\operatorname{Im}\left\{\widetilde{\Theta}_{G}, \mathcal{O} \otimes W_{n, 2}\right\} \otimes \mathcal{O}(-1)$ is $\mathcal{O}(-n+1)$. This immediately implies the assertion. Note here that the summand $\mathcal{O}(-n+1)$ is the kernel of the operator $\widetilde{\Theta}_{G}$ on the subspace generated by $v_{1}, \ldots, v_{n}$ (in the notation of Definition 3.1).

The extension of Proposition 6.3 to all $W_{n, d}$ now follows as we show in our next proposition.

Proposition 6.4. As in Proposition 6.3, we view the $\mathbb{Z} / p \times \mathbb{Z} / p$-module $W_{n, d}$ as a $G=\mathbb{G}_{a(1)} \times \mathbb{G}_{a(1)}$-module. Then

$$
\operatorname{Ker}\left\{\widetilde{\Theta}_{G}, \mathcal{O} \otimes W_{n, d}\right\} \simeq \mathcal{O}^{\oplus n+1-d} \oplus \bigoplus_{i=1}^{d-1} \mathcal{O}(-n+i)
$$

Proof. The proposition is proved by an induction on Loewy length $d$, using the embedding $\iota_{n, d}: W_{n-1, d-1} \hookrightarrow W_{n, d}$ of (4). By induction, $\operatorname{Ker}\left\{\widetilde{\Theta}_{G}, \mathcal{O} \otimes W_{n-1, d-1}\right\}$ is isomorphic to $\mathcal{O}^{\oplus n+1-d} \oplus \bigoplus_{i=1}^{d-2} \mathcal{O}(-n+i)$ and embeds in $\operatorname{Ker}\left\{\widetilde{\Theta}_{G}, \mathcal{O} \otimes W_{n, d}\right\}$. The summand $\mathcal{O}(-n+1)$ of $\operatorname{Ker}\left\{\widetilde{\Theta}_{G}, \mathcal{O} \otimes W_{n, 2}\right\}$ arising in Proposition 6.3 comes from the kernel of the operator $\Theta_{G}$ on the first layer (i.e., the head) of $k[s, t] \otimes W_{n, 2}$. Since multiplication by $\Theta_{G}$ on $k[s, t] \otimes W_{n, d}$ can be viewed as an endomorphism of degree -1 of this $k[s, t]$-module equipped with a grading provided by $\Theta_{G}$, we conclude that this summand $\mathcal{O}(-n+1)$ of $\operatorname{Ker}\left\{\widetilde{\Theta}_{G}, \mathcal{O} \otimes W_{n, 2}\right\}$ embeds in $W_{n, d}$ in such a way that its intersection with $\operatorname{Ker}\left\{\widetilde{\Theta}_{G}, \mathcal{O} \otimes W_{n-1, d-1}\right\}$ is trivial. Thus, we have a natural injective map

$$
\mathcal{O}(-n+1) \oplus \operatorname{Ker}\left\{\widetilde{\Theta}_{G}, \mathcal{O} \otimes W_{n-1, d-1}\right\} \rightarrow \operatorname{Ker}\left\{\widetilde{\Theta}_{G}, \mathcal{O} \otimes W_{n, d}\right\} .
$$

We readily verify that $\operatorname{Ker}\left\{\widetilde{\Theta}_{G}, \mathcal{O} \otimes W_{n, d}\right\}$ has rank $n$. That is, the homomorphism $\Theta_{G}: k[s, t] \otimes W_{n, d} \rightarrow k[s, t] \otimes W_{n, d}$ maps the $i^{\text {th }}$ layer of $W_{n, d}$ (which has rank $n+1-i)$ onto the $(i-1)^{s t}$ layer for $i<d$ and it maps the $d^{\text {th }}$ layer to 0 . Consequently, we conclude that (16) is an isomorphism by a comparison of dimensions.

The computation of the kernel of

$$
\widetilde{\Theta}_{G}^{2}=\widetilde{\Theta}_{G} \circ \widetilde{\Theta}_{G}: \mathcal{O} \otimes W_{n, d} \rightarrow \mathcal{O}(2) \otimes W_{n, d}
$$

now follows easily since Serre's computation of the cohomology of $\mathbb{P}^{1}$ (see [9], III.5.1) implies that the extension mentioned below is split. 
Proposition 6.5. Let $G=\mathbb{G}_{a(1)} \times \mathbb{G}_{a(1)}$. We regard $W_{n, d}$ as a $k G$-module, via the isomorphism of the group algebra of $\mathbb{Z} / p \times \mathbb{Z} / p$ with $k G$. Then

$\operatorname{Ker}\left\{\widetilde{\Theta}_{G}^{2}, \mathcal{O} \otimes W_{n, d}\right\} \simeq \operatorname{Ker}\left\{\widetilde{\Theta}_{G}, \mathcal{O} \otimes W_{n, d}\right\} \oplus \operatorname{Ker}\left\{\widetilde{\Theta}_{G}, \mathcal{O} \otimes W_{n-1, d-1}\right\}$

$$
\simeq \mathcal{O}^{\oplus 2 n+2-2 d} \oplus\left(\bigoplus_{i=2}^{d-1} \mathcal{O}(-n+i)^{\oplus 2}\right) \oplus \mathcal{O}(-n+1) .
$$

Proof. The second asserted isomorphism follows immediately from Proposition 6.4.

To establish the first isomorphism, we investigate $\operatorname{Ker}\left\{\widetilde{\Theta}_{G}^{2}, \mathcal{O} \otimes W_{n, d}\right\}$ by observing that it fits in a natural short exact sequence

$$
\begin{aligned}
0 \longrightarrow & \operatorname{Ker}\left\{\widetilde{\Theta}_{G}, \mathcal{O} \otimes W_{n, d}\right\} \longrightarrow \operatorname{Ker}\left\{\widetilde{\Theta}_{G}^{2}, \mathcal{O} \otimes W_{n, d}\right\} \\
& \longrightarrow \operatorname{Ker}\left\{\widetilde{\Theta}_{G}, \operatorname{Im}\left\{\widetilde{\Theta}_{G}, \mathcal{O} \otimes W_{n, d}\right\}\right\} \longrightarrow 0 .
\end{aligned}
$$

As argued in the proof of Proposition 6.4, multiplication by $\Theta_{G}$ on $k[s, t] \otimes W_{n, d}$ can be viewed as an endomorphism of degree -1 of this $k[s, t]$-module equipped with a grading provided by $\Theta_{G}$. Thus, we have the identification

$$
\operatorname{Ker}\left\{\widetilde{\Theta}_{G}^{2}, \mathcal{O} \otimes W_{n, d}\right\} \simeq \bigoplus_{i=1}^{d} K(i),
$$

where

$$
\begin{aligned}
K(i)=\operatorname{Ker}\left\{\widetilde{\Theta}_{G}^{2}: \mathcal{O} \otimes\right. & W_{n-i, d-i} \\
& \left.\rightarrow\left(\mathcal{O}(2) \otimes W_{n-i-2, d-i-2}\right) /\left(\mathcal{O}(2) \otimes W_{n-i-3, d-i-3}\right)\right\} .
\end{aligned}
$$

Restricting the extension (17) to $K(i)$ we obtain extensions

$$
\begin{aligned}
0 \longrightarrow & \operatorname{Ker}\left\{\widetilde{\Theta}_{G}, \mathcal{O} \otimes W_{n-i, d-i}\right\} \longrightarrow K(i) \\
& \longrightarrow \operatorname{Ker}\left\{\widetilde{\Theta}_{G}, \mathcal{O} \otimes W_{n-i-1, d-i-1}\right\} \longrightarrow 0 .
\end{aligned}
$$

The proposition now follows from the observation that the extensions (18) split, since the corresponding coherent $\mathrm{Ext}^{1}$ groups vanish thanks to Serre's computation of $\mathrm{H}^{*}\left(\mathbb{P}^{1}, \mathcal{O}(\ell)\right)$. The one non-vanishing Ext ${ }^{1}$ group (of coherent $\mathcal{O}$-modules) which arises in this extension occurs in the case $n=3$, with $\operatorname{Ext}_{\mathcal{O}}^{1}(\mathcal{O}, \mathcal{O}(-3+1)) \neq 0$. For this one case, one easily observes that the copy of $\mathcal{O}$ in the quotient splits.

\section{Generic kernels}

Throughout this section, we assume that the field $k$ is infinite. We continue to use the notation $\mathbb{P}^{1}(k)$ to denote the $k$-rational points of $\mathbb{P}^{1}$ which we identify with the 
collection of lines in $k^{2}$. That is, $\mathbb{P}^{1}(k)$ is the set of equivalence classes $\langle a, b\rangle$ of pairs $(0,0) \neq(a, b) \in k^{2}$, where two pairs are equivalent if they lie on the same line through the origin. We associate to any (finite dimensional) $k G$-module $M$ a $k G$ submodule $\mathbb{R}(M) \subset M$ which has constant Jordan type. Indeed, the characterization in Theorem 7.10 of $\Omega(M) \subset M$ as the maximal submodule of $M$ which has the equal images property implies that $M \mapsto \Omega(M)$ is functorial. This is not immediately evident granted the choices we make in Definition 7.1.

We fix a choice of generators $x, y$ of $\operatorname{Rad}(k G)$, so that $k G \simeq k[x, y] /\left(x^{p}, y^{p}\right)$. For any $\langle a, b\rangle \in \mathbb{P}^{1}(k)$, write

$$
\langle a, b\rangle=\operatorname{Ker}\{a x+b y: M \rightarrow M\} .
$$

Note that $\langle a, b\rangle M$ does not depend on the choice of the representing pair $(a, b) \in k^{2}$.

For any subset $S \subset \mathbb{P}^{1}(k)$, define the subspace

$$
{ }_{S} M=\sum_{\langle a, b\rangle \in S}\langle a, b\rangle M
$$

Since both $x$ and $y$ commute with each $a x+b y \in S,{ }_{S} M$ is a $k G$-submodule of $M$. Clearly, for $S^{\prime} \subset S, S^{\prime} M \subset S M$.

In the degenerate case in which $S=\emptyset$, we set ${ }_{S} M=\{0\}$.

Definition 7.1. Let $k$ be an infinite field and let $M$ be a finite dimensional $k G$-module. We define the generic kernel of $M$ to be

$$
\Re(M)=\bigcap_{S \subset \mathbb{P}^{1}(k) \text { cofinite }} S M .
$$

Because $M$ is finite dimensional, we may choose for a given $M$ a cofinite $S \subset$ $\mathbb{P}^{1}(k)$ such that $S M=\Omega(M)$. Observe that

$$
\Re(\Re(M))=\Re(M),
$$

since $T(S M)=S \cap T M$.

We give the following elementary example. More examples follow later.

Example 7.2. Let $M=k G$, the free cyclic $k G$-module, with $k$ infinite. Then

$$
\Re(M)=\operatorname{Rad}^{p-1}(k G) \simeq W_{p, p} .
$$

To prove the equality $\Omega(M)=\operatorname{Rad}^{p-1}(k G)$, we first verify that $\Omega(M)$ is contained in $\operatorname{Rad}^{p-1}(k G)$. For this we note that $k G$ has constant Jordan type $p[p]$ and hence any element of $\operatorname{Ker}\{a x+b y: M \rightarrow M\}$ must be in $(a x+b y)^{p-1} k G \subseteq \operatorname{Rad}^{p-1}(k G)$. Hence, $\Omega(k G) \subseteq \operatorname{Rad}^{p-1}(k G)$ by the definition. On the other hand, for any $a, b \in k$, 
$(a x+b y)^{p-1} \in \operatorname{Ker}\{a x+b y: M \rightarrow M\}$. Hence, for any cofinite set $S \subseteq \mathbb{P}^{1}$, the elements $(a x+b y)^{p-1}$ with $a x+b y$ in $S$ generate all of $\operatorname{Rad}^{p-1}(k G)$ (see Proposition 3.6). So, $\operatorname{Rad}^{p-1}(k G) \subseteq \Re(k G)$ by the definition of the generic kernel.

It was shown in Example 3.2 that $\operatorname{Rad}^{p-1}(k G) \simeq W_{p, p}$ has the equal images property and thus is equal to its own generic kernel. Hence, the calculations in the examples could also be verified using Proposition 7.8 which follows later in the section.

Proposition 7.3. Suppose that the field $k$ is algebraically closed. Let $M$ be any $k G$-module. Then $\Omega(M)$ has the equal images property.

Proof. Using (19), we may and do assume that $M=\Re(M)$. By Proposition 2.6, it suffices to show for $(0,0) \neq(a, b) \in k^{2}$ that $\ell_{(a, b)} \cdot M=x \cdot M$. (In this notation, $\ell_{(1,0)} M=x \cdot M$.) Clearly, we may assume that $b \neq 0$. Write $M={ }_{S} M$ where $S \subseteq \mathbb{P}^{1}$ is cofinite and $S$ contains neither $\langle 1,0\rangle$ (which corresponds to $x \in \operatorname{Rad}(k G)$ ) nor $\langle a, b\rangle$. For any $\langle c, d\rangle \in S$, there exist elements $e, f \in k$ such that $c x+d y=$ $e x+f(a x+b y)$. Note here that $e$ and $f$ are both not zero because the three points $\langle 1,0\rangle,\langle c, d\rangle,\langle a, b\rangle$ are distinct. Then

$$
\ell_{(1,0)} \cdot\langle c, d\rangle=\ell_{(a, b)} \cdot\langle c, d\rangle M .
$$

The proposition now follows from the definition of the generic kernel by taking the sum indexed by $\langle c, d\rangle \in S$ on both sides of (20).

The following proposition justifies our definition of generic kernel without passing to the algebraic closure of our base field $k$.

Proposition 7.4. Let $k$ be an infinite field and $M$ a $k G$-module. Then for any field extension $L / k$,

$$
L \otimes \Re(M)=\Re\left(M_{L}\right) .
$$

Proof. Let $S \subset \mathbb{P}^{1}(k)$ be a cofinite subset such that $\Omega(M)={ }_{S} M$. We first assume that the field $L$ is algebraically closed. By Proposition 7.3, $\Omega\left(M_{L}\right)$ has the equal images property so that we may apply Corollary 5.5 to $\Omega\left(M_{L}\right)$ and the infinite subset $S \subset \mathbb{P}^{1}(K) \subset \mathbb{P}^{1}(L)$. We conclude that

$$
\begin{aligned}
\Re\left(M_{L}\right) & =\sum_{\langle a, b\rangle \in S} \operatorname{Ker}\left\{a x+b y: \Omega\left(M_{L}\right) \rightarrow \Omega\left(M_{L}\right)\right\} \\
& =L \otimes \sum_{\langle a, b\rangle \in S} \operatorname{Ker}\{a x+b y: \Omega(M) \rightarrow \Omega(M)\}=L \otimes \Omega(M) .
\end{aligned}
$$


For an arbitrary field extension $L / k$, let $T \subset \mathbb{P}^{1}(L)$ be a cofinite subset such that $\mathscr{R}\left(M_{L}\right)={ }_{T}\left(M_{L}\right)$. Since $S \cap T$ is also cofinite in $\mathbb{P}^{1}(k)$, we may assume that $S=S \cap T$. Thus,

$$
L \otimes \Omega(M)=L \otimes{ }_{S} M=\sum_{\langle a, b\rangle \in S} L \otimes\langle a, b\rangle=\sum_{\langle a, b\rangle \in S}\langle a, b\rangle\left(M_{L}\right) \subseteq \Re\left(M_{L}\right) .
$$

Let $\bar{L}$ be the algebraic closure of $L$. By the first part of this proof, we have that

$$
\operatorname{Dim}_{k}(\Omega(M))=\operatorname{Dim}_{\bar{L}}(\Omega(\bar{L} \otimes M))=\operatorname{Dim}_{L}\left(\Omega\left(M_{L}\right)\right) .
$$

Since $L \otimes \Re(M) \subseteq \Re\left(M_{L}\right),(21)$ implies the asserted equality $L \otimes \Re(M)=\Re\left(M_{L}\right)$.

We now extend Proposition 7.3 to arbitrary infinite fields.

Proposition 7.5. Let $k$ be an infinite field and $M$ a finite dimensional $k G$-module. The generic kernel of $M$ has the equal images property. In particular, $\mathbb{R}(M)$ has constant Jordan type.

Proof. Let $\Omega / k$ be an algebraic closure. By Proposition 7.3, $\Omega\left(M_{\Omega}\right)$ has the equal images property. By the preceding Proposition 7.4, this is equivalent to the assertion that $\Omega \otimes \Omega(M)$ has the equal images property. By the equivalence of (1) and (2) of Proposition 2.6, this implies that $\Omega(M)$ has the equal images property.

The second assertion follows immediately from the first and Proposition 2.8.

We next verify that the maximal subset $S \subset \mathbb{P}^{1}(k)$ with the property that ${ }_{S} M=$ $\mathscr{R}(M)$ has a natural characterization in terms of the action of $k G$ on $M$. Observe that any infinite subset $S \subset \mathbb{P}^{1}(k)$ is dense in $\mathbb{P}^{1}$; in other words, any non-empty open subset $U \subset \mathbb{P}^{1}$ contains a point of $S$.

Proposition 7.6. Let $k$ be an infinite field and $M$ a finite dimensional $k G$-module with generic kernel $\Omega(M)$. Let $r$ be the maximum of the ranks $\operatorname{Rank}\left\{\alpha_{K}(t): M \rightarrow M\right\}$ for all $\pi$-points $\alpha_{K}: K[t] /\left(t^{p}\right) \rightarrow K G$. Then we have that

(1) for any $\ell_{(a, b)}=a x+b y,(a, b) \neq(0,0), a, b \in k$, the kernel of the multiplication $\operatorname{Ker}\left\{\ell_{(a, b)}: M \rightarrow M\right\}$ is a subset of $\Omega(M)$ if and only if the rank of the operator $\ell_{(a, b)}: M \rightarrow M$ is $r$, and

(2) the cofinite subset

$$
\left\{\langle a, b\rangle \mid \operatorname{Rank}\left\{\ell_{(a, b)}: M \rightarrow M\right\}=r\right\} \subset \mathbb{P}^{1}(k)
$$

is the largest subset $S \subset \mathbb{P}^{1}(k)$ with the property that ${ }_{S} M=\Re(M)$. 
Proof. First note that the set of all classes of $\pi$-points $\alpha_{K}: K[t] /\left(t^{p}\right) \rightarrow k G$ with the rank of $\alpha_{K}(t)$ maximal on $M_{K}$ is an open subset of $\Pi(G)$ by [7]. Hence, there is some $\langle c, d\rangle \in \mathbb{P}^{1}(k)$ such that $\operatorname{Rank}\left\{\ell_{\langle c, d\rangle}: M \rightarrow M\right\}$ is maximal and satisfies the condition that $\operatorname{Ker}\left\{\ell_{\langle c, d\rangle}: M \rightarrow M\right\} \subset \Re(M)$. For an arbitrary $\langle a, b\rangle$ in $\mathbb{P}^{1}(k)$, the inclusion $\operatorname{Ker}\left\{\ell_{\langle a, b\rangle}: \Omega(M) \rightarrow \Omega(M)\right\} \hookrightarrow \operatorname{Ker}\left\{\ell_{\langle a, b\rangle}: M \rightarrow M\right\}$ is the identity if and only if $\operatorname{Ker}\left\{\ell_{\langle a, b\rangle}: M \rightarrow M\right\} \subset \Re(M)$.

We have the following relations:

$$
\begin{aligned}
\operatorname{Dim}\left(\operatorname{Ker}\left\{\ell_{\langle a, b\rangle}: M \rightarrow M\right\}\right) & \geq \operatorname{Dim}\left(\operatorname{Ker}\left\{\ell_{\langle c, d\rangle}: M \rightarrow M\right\}\right) \\
& =\operatorname{Dim}\left(\operatorname{Ker}\left\{\ell_{\langle c, d\rangle}: \Re(M) \rightarrow \Re(M)\right\}\right) \\
& =\operatorname{Dim}\left(\operatorname{Ker}\left\{\ell_{\langle a, b\rangle}: \Re(M) \rightarrow \Omega(M)\right\}\right) .
\end{aligned}
$$

In the above chain, the inequality is a consequence of the maximality of the rank of $\ell_{\langle c, d\rangle}$, the middle equality follows from the choice of $\ell_{\langle c, d\rangle}$ and the preceding observation, and the last equality is a consequence of the equal images property of $\Omega(M)$. Thus, we conclude that the left inequality is an equality (and thus $\ell_{\langle a, b\rangle}$ has maximal rank) if and only if $\operatorname{Ker}\left\{\ell_{\langle a, b\rangle}: M \rightarrow M\right\}=\operatorname{Ker}\left\{\ell_{\langle a, b\rangle}: \mathscr{R}(M) \rightarrow\right.$ $\Re(M)\}$. This happens if and only if $\operatorname{Ker}\left\{\ell_{\langle a, b\rangle}: M \rightarrow M\right\} \subset \Re(M)$, by another application of the above observation.

If $\ell_{\left\langle a^{\prime}, b^{\prime}\right\rangle}: M \rightarrow M$ does not have maximal rank and if $T \subset \mathbb{P}^{1}(k)$ contains $\left\langle a^{\prime}, b^{\prime}\right\rangle$, then $\operatorname{Ker}\left\{\ell_{\left\langle a^{\prime}, b^{\prime}\right\rangle}^{\prime}: M \rightarrow M\right\} \subset{ }_{T} M$, so that ${ }_{T} M$ is not contained in $\Omega(M)$.

As an immediate corollary of Proposition 7.6, we obtain the following characterization of a $k G$-module of constant rank.

Corollary 7.7. Let $k$ be an infinite field and $M$ a finite dimensional $k G$-module. Then $\Omega(M)={ }_{\mathbb{P}^{1}(k)} M$ if and only if the rank of $\ell_{(a, b)}$ on $M$ is independent of $\langle a, b\rangle \in \mathbb{P}^{1}(k)$.

Proposition 7.8. We have the following.

(1) Suppose that $\psi: M \rightarrow N$ is a homomorphism of $k G$-modules. Then we have that $\psi(\Omega(M)) \subseteq \Omega(N)$.

(2) If $M$ is a $k G$-module having the equal images property, then $\Omega(M)=M$. In particular $\Re\left(W_{n, d}\right)=W_{n, d}$ for any $n$ and $d$.

(3) The association of a finite dimensional $k G$-module $M$ to its generic kernel determines a functor

$$
\Re: \bmod (k G) \rightarrow \bmod (k G) .
$$

Proof. The first statement is a consequence of the observation that

$$
\psi(\operatorname{Ker}\{\ell: M \rightarrow M\}) \subseteq \operatorname{Ker}\{\ell: N \rightarrow N\},
$$


for any $\ell \in k G$. Hence, if $\Omega(N)={ }_{S} N$ for $S$ cofinite in $\mathbb{P}^{1}(k)$, then

$$
\psi(\Omega(M)) \subseteq \psi(s M) \subseteq{ }_{S} N=\Omega(N) .
$$

This proves the first statement. The second part follows from Proposition 3.6 and Corollary 5.5. The third statement is obvious from the first two.

Clearly, the functor $\Omega(-)$ preserves monomorphisms. However, as the following example shows, $\mathscr{R}(-)$ is not left exact. (The augmentation map $k G \rightarrow k$ is a simple example of an epimorphism with the property that the induced map on generic kernels is not an epimorphism.)

Example 7.9. Let $M=\operatorname{Rad}^{p-1}(k G)$ and let $L \subseteq M$ be the submodule generated by $x^{p-1}$. Then we have an exact sequence

$$
0 \longrightarrow L \longrightarrow M \longrightarrow N \longrightarrow 0
$$

where $N=M / L$ is the quotient. The modules $M$ and $N$ have the equal images property and hence they are equal to their own generic kernels. However, it is easy to check that the generic kernel of $L$ is its socle which is isomorphic to $k$. So applying the functor $\Omega$ we obtain the sequence

$$
0 \longrightarrow k \longrightarrow M \longrightarrow N
$$

which is not exact at $M$.

The following theorem characterizes the generic kernel $\Omega(M)$ of $M$.

Theorem 7.10. Let $M$ be a finite dimensional $k G$-module. The generic kernel $\Omega(M)$ of $M$ contains every submodule of $M$ having the equal images property. Thus, $\Omega(M)$ is the maximal submodule of $M$ which has the equal images property. Moreover, $\Omega(M)$ is the maximal submodule of $M$ which can be written as the quotient of a $W$ module.

Proof. The first two assertions are consequences of Propositions 7.3 and 7.8. The last follows from Proposition 5.4.

We obtain as an immediate corollary a proof of the statement that $\Omega(M) \subset M$ is "intrinsic".

Corollary 7.11. Let $M$ be a finite dimensional $k G$-module. Then $\Omega(M)$ does not depend upon the choice of the generators of $\operatorname{Rad}(k G)$.

We explicitly determine generic kernels of the syzygy modules $\Omega^{n}(k)$ in Example 7.12 and " $L_{\zeta}$-modules" in Example 7.13. 
Example 7.12. A minimal projective resolution of the trivial module $k$ for $k G$ can be given as

$$
\cdots \longrightarrow P_{2} \stackrel{\partial_{2}}{\longrightarrow} P_{1} \stackrel{\partial_{1}}{\longrightarrow} P_{0} \stackrel{\varepsilon}{\longrightarrow} k \longrightarrow 0
$$

where $P_{n} \simeq(k G)^{n+1}$ is a free $k G$-module with free basis $c_{n, 0}, \ldots, c_{n, n}$. The boundary maps are given by the formulae

$$
\begin{gathered}
\partial_{2 n-1}\left(c_{2 n-1,0}\right)=x c_{2 n-2,0}, \\
\partial_{2 n-1}\left(c_{2 n-1,2 i-1}\right)=x^{p-1} c_{2 n-2,2 i-1}+y c_{2 n-2,2 i-2}, \\
\partial_{2 n-1}\left(c_{2 n-1,2 i}\right)=x c_{2 n-2,2 i}-y^{p-1} c_{2 n-2,2 i-1}, \\
\partial_{2 n-1}\left(c_{2 n-1,2 n-1}\right)=y c_{2 n-2,2 n-2},
\end{gathered}
$$

and

$$
\begin{gathered}
\partial_{2 n}\left(c_{2 n, 0}\right)=x^{p-1} c_{2 n-1,0}, \\
\partial_{2 n}\left(c_{2 n, 2 i-1}\right)=x c_{2 n-1,2 i-1}-y c_{2 n-1,2 i-2}, \\
\partial_{2 n}\left(c_{2 n, 2 i}\right)=x^{p-1} c_{2 n-1,2 i}+y^{p-1} c_{2 n-1,2 i-1}, \\
\partial_{2 n}\left(c_{2 n, 2 n}\right)=y^{p-1} c_{2 n-1,2 n-1} .
\end{gathered}
$$

This is a standard, well-known calculation [3].

From the above we get that the module $\Omega^{2 n-1}(k)$ has generators $a_{1}, b_{1}, \ldots, a_{n}, b_{n}$ and relations

$$
x^{p-1} a_{1}=0=y^{p-1} b_{n}, \quad y a_{i}=x b_{i}, \quad \text { and } \quad x^{p-1} a_{i}=-y^{p-1} b_{i-1} .
$$

That is, we take $a_{i}$ to be the class of $c_{2 n-1,2 i}$ modulo the image of $\partial_{2 n}$ and $b_{i}$ to be the class of $c_{2 n-1,2 i-1}$. The module $\Omega^{2 n}(k)$ is generated by elements $a_{1}, b_{1}, a_{2}, \ldots, a_{n}$, $b_{n}, a_{n+1}$ with relations

$$
x a_{1}=0=y a_{n+1}, \quad x a_{i}=y^{p-1} b_{i-1}, \quad \text { and } \quad y a_{i}=-x^{p-1} b_{i} .
$$

In the case that $p=3$, we can draw diagrams which reveal the structure of $\Omega^{3}(k)$ and $\Omega^{4}(k)$ as follows:

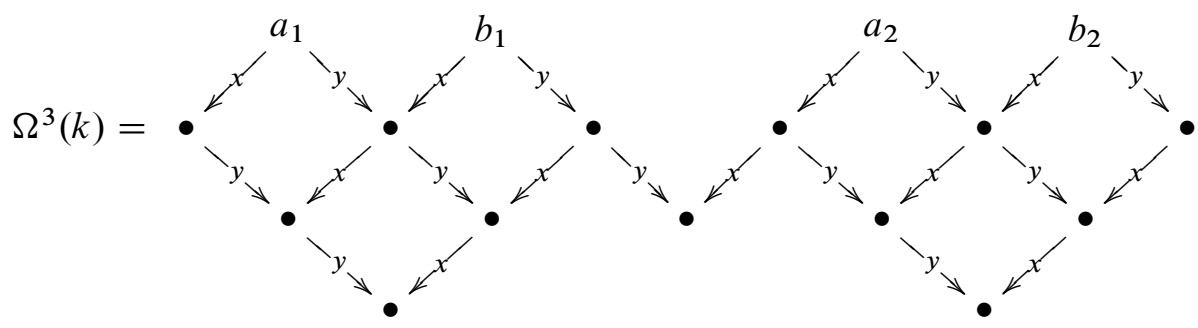


and

$$
\Omega^{4}(k)=
$$

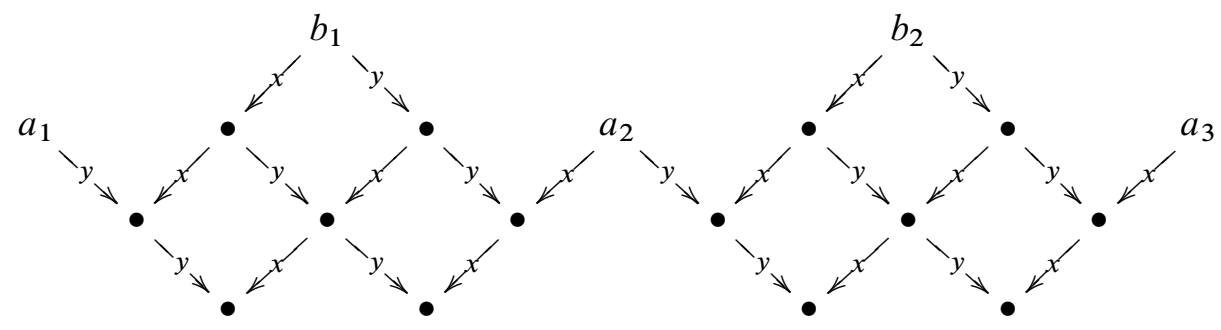

With the above information, it can be calculated that $\Omega\left(\Omega^{2 n-1}(k)\right)$ is the submodule of $\Omega^{2 n-1}(k)$ generated by the elements

$$
x^{j} y^{p-2-j} a_{i}, \quad x^{j} y^{p-2-j} b_{i} \text { for } j=0, \ldots, p-2 \text { and } i=1, \ldots, n .
$$

Likewise, we have that $\Omega\left(\Omega^{2 n}(k)\right)$ is the submodule generated by the elements

$$
\begin{gathered}
a_{1}, \ldots, a_{n+1}, \quad \text { and } \\
x^{j} y^{p-2-j} b_{i}, \quad \text { for } j=0, \ldots, p-2 \text { and } i=1, \ldots, n .
\end{gathered}
$$

The above conclusion is made using Theorem 7.10. That is, we need only look for the largest submodule having the equal images property. In the diagrams we look for the largest submodule having the "W" shape. This exercise is left to the reader.

Example 7.13. Similar arguments can be made for the $L_{\zeta}$ modules. For example, suppose that $\zeta \in \mathrm{H}^{n}(G, k)$ is a nilpotent element. Let $L_{\zeta}$ be the module which is the kernel of a cocycle

$$
\zeta: \Omega^{n}(k) \rightarrow k,
$$

representing the cohomology element $\zeta$. Then $\Omega\left(L_{\zeta}\right)=\Omega\left(\Omega^{n}(k)\right)$, the isomorphism being induced by the inclusion of $L_{\zeta}$ into $\Omega^{n}(k)$.

A justification for the above statement is the following. Suppose that $\ell=a x+b y$ for $(a, b) \neq(0,0)$ in $k^{2}$. If $n$ is even, then the restriction $\Omega^{n}(k)_{\downarrow\langle\ell\rangle}$ of $\Omega^{n}(k)$ to the subalgebra generated by $\ell$ has the form $k \oplus k\langle\ell\rangle^{s}$ for some $s$. Here $k\langle\ell\rangle$ is the rank one free module over $k\langle\ell\rangle \cong k[t] /\left(t^{p}\right)$. Because $\zeta$ is nilpotent, we must have that the summand isomorphic to $k$ lies in the kernel of the cocyle $\zeta$, as otherwise the cocycle $\zeta$ would be left split. In particular,

$$
\operatorname{Ker}\left\{\ell: \Omega^{n}(k) \rightarrow \Omega^{n}(k)\right\} \subseteq \operatorname{Ker}\{\zeta\} .
$$

It is not difficult to see that the same happens also in the case that $n$ is odd. Hence, from the definition of the generic kernel, we can see that $\Omega\left(\Omega^{n}(k)\right) \subseteq \operatorname{Ker}\{\zeta\}=L_{\zeta}$, as asserted. 
On the other hand, if $\zeta$ not nilpotent, then there exists an open subset $S$ of $\mathbb{P}^{1}$, such that for any $\langle a, b\rangle \in S$, the summand isomorphic to $k$ in the decomposition of the restriction of $\Omega^{n}(k)$ to $\left\langle\ell_{(a, b)}\right\rangle$ is not in the kernel of $\zeta$. As a result $\Omega\left(L_{\zeta}\right)$ is a proper submodule of $\Omega\left(\Omega^{n}(k)\right)$. Again we should look for modules with the "W" shape. It can be proved in a similar way that for $\zeta$ not nilpotent, $\mathscr{\Omega}\left(L_{\zeta}\right)=x \Re\left(\Omega^{n}(k)\right)=$ $\operatorname{Rad}\left(\Omega\left(\Omega^{n}(k)\right)\right)$.

\section{Duality and filtrations}

The purpose of this section is to show that some of the objects defined earlier in the paper have dual notions. The main result of the section shows that the generic kernel, together with its dual, the generic image, defines a filtration on any module of constant Jordan type. The filtration is distinct from the radical filtration and invariant under automorphisms of the group algebra.

In this section we assume that the field $k$ is infinite. Again, we denote the set of lines through the origin in $k^{2}$ by $\mathbb{P}^{1}(k)$, the set of $k$-rational points of $\mathbb{P}^{1}$. Let $k G$ be the group algebra of an elementary abelian group of rank 2 , and fix generators $x$ and $y$ of the radical of $k G$. For $M$ a finite dimensional $k G$-module, we let $M^{\#}=$ $\operatorname{Hom}_{k}(M, k)$ denote its $k$-linear dual. Recall that $M^{\#}$ is a $k G$-module under the operation defined by the equation $(g f)(m)=f\left(g^{-1} m\right)$ where $g \in G, f \in M^{\#}$ and $m \in M$. For each $V \subset M$, we associate

$$
V^{\perp}=\{f: M \rightarrow k \mid f(V)=0\} \subset M^{\#} .
$$

Note that if $V$ is a $k G$-submodule of $M$, then $V^{\perp}$ is a submodule of $M^{\#}$.

Lemma 8.1. Let $M$ be a finite dimensional $k[t] /\left(t^{p}\right)$-module and let $V \subset M$ be a submodule. Then the Jordan type of $V$ is the same as that of $M^{\#} / V^{\perp}$.

Proof. The canonical pairing

$$
V \otimes\left(M^{\#} / V^{\perp}\right) \rightarrow k, \quad v \otimes f \mapsto f(v)
$$

is a perfect (non-degenerate) pairing of $k[t] /\left(t^{p}\right)$-modules. The lemma follows by observing that the $k[t] /\left(t^{p}\right)$-dual $W^{\#}$ of a $k[t] /\left(t^{p}\right)$-module $W$ has the same Jordan type as that of $W$.

We introduce the generic image $\mathfrak{I}(M)$ of a finite dimensional $k G$-module $M$, whose construction and properties are analogous to those of the generic kernel $\Omega(M)$ of $M$. We see in Proposition 8.4 that $\mathscr{R}(M)$ and $\mathfrak{I}(M)$ are related by duality. 
For any cofinite subset $S \subset \mathbb{P}^{1}(k)$, define the subspace

$$
M_{S}=\bigcap_{\langle a, b\rangle \in S} \operatorname{Im}\{a x+b y: M \rightarrow M\}
$$

in analogy with ${ }_{S} M$ of the last section. Here, as before, the image of multiplication by $\ell=a x+b y$ is independent of the representative of the class $\langle a, b\rangle \in \mathbb{P}^{1}$.

Since both $x, y$ commute with each $a x+b y$ for $\langle a, b\rangle \in S, M_{S}$ is a $k G$ submodule of $M$. Clearly, for $S^{\prime} \subset S, M_{S^{\prime}} \supset M_{S}$.

Definition 8.2. We define the generic image of $M$ to be

$$
\mathfrak{s}(M)=\sum_{S \subset \mathbb{P}^{1}(k) \text { cofinite }} M_{S}
$$

As with the generic kernel, because $M$ is finite dimensional, there exists a subset $S$ in $\mathbb{P}^{1}(k)$ which is cofinite and has the property that $M_{S}=\mathfrak{s}(M)$.

Example 8.3. Suppose that $M$ is the submodule of $k G$ generated by $m=y^{p-1}$. Then $(a x+b y) m=a x m$. Consequently, $\mathfrak{s}(M)=\operatorname{Rad}(k G) M=\operatorname{Rad}(M)$. On the other hand, the only subspace of $M$ which is annihilated by $(a x+b y)$ for $a \neq 0$ is generated by $x^{p-1} m$. Hence $\Omega(M)$ is the submodule $x^{p-1} M$, which is strictly contained in $\mathfrak{I}(M)$. This situation contrasts sharply with the results of Theorem 8.6 that follows. We note also that $\mathfrak{I}(M)$ does not have constant rank in this example.

Proposition 8.4. Let $M$ be a finitely generated $k G$-module. Then

$$
\mathfrak{I}(M) \simeq \Re\left(M^{\#}\right)^{\perp}, \quad \Re(M) \simeq \mathfrak{I}\left(M^{\#}\right)^{\perp} .
$$

It follows that $M^{\#} / \mathfrak{I}\left(M^{\#}\right)$ has constant Jordan type. In addition, $\mathfrak{I}(M)$ has constant Jordan type if and only if $M^{\#} / \Omega\left(M^{\#}\right)$ does, in which case their Jordan types are equal.

Proof. For any linear map $\ell: M \rightarrow M$,

$$
(\ell M)^{\perp}=\{g: M \rightarrow k \mid g \circ \ell=0\}=\operatorname{Ker}\left\{\ell^{\#}: M^{\#} \rightarrow M^{\#}\right\}
$$

For every cofinite subset $S \subset \mathbb{P}^{1}(k)$,

$$
\left(\bigcap_{\langle a, b\rangle \in S} \operatorname{Im}\{a x+b y: M \rightarrow M\}\right)^{\perp}=\sum_{\langle a, b\rangle \in S} \operatorname{Ker}\left\{(a x+b y)^{\#}: M^{\#} \rightarrow M^{\#}\right\} .
$$

Thus, we conclude that

$$
\mathfrak{I}(M)^{\perp}=\Re\left(M^{\#}\right) .
$$


It follows that

$$
M / \mathfrak{I}(M) \simeq \mathfrak{I}\left(M^{\#}\right)^{\perp}=\Omega(M) \text { and } \mathfrak{I}(M)=\Omega\left(M^{\#}\right)^{\perp} \simeq M / \Omega(M) .
$$

The second statement is a consequence of the fact that $\Omega(M)$ has the equal images property.

The next lemma is key to the proof of Theorem 8.6.

Lemma 8.5. Let $M$ be a $k G$-module of constant rank. Then for any $\langle a, b\rangle \in \mathbb{P}^{1}(k)$, the kernel of multiplication by ax + by on $M / x \Re(M)$ is equal to $\mathbb{R}(M) / x \Re(M)$. Hence, $(M / x \Re(M))^{\#} \subseteq M^{\#}$ has the equal images property, and thus

$$
(M / x \Omega(M))^{\#} \subseteq \Omega\left(M^{\#}\right) .
$$

Proof. Let $\bar{M}$ denote $M / x \Omega(M)$. By Corollary 7.7, we know that $\Omega(M)={ }_{S}(M)$ where $S=\mathbb{P}^{1}$. Observe that

$$
\Re(M) / x \Re(M) \subset \operatorname{Ker}\{a x+b y: \bar{M} \rightarrow \bar{M}\},
$$

since we know that $(a x+b y) \Re(M)=x \Re(M)$.

To prove the reverse inclusion, suppose that $m+\Omega(M)$ is in $\operatorname{Ker}\{a x+b y: \bar{M} \rightarrow$ $\bar{M}\}$ for some $m$ in $M$. Then $(a x+b y) m \in x \Re(M)=(a x+b y) \Re(M)$. So $(a x+b y) m=(a x+b y) m^{\prime}$ for some $m^{\prime} \in \Omega(M)$. But then $(a x+b y)\left(m-m^{\prime}\right)=0$ and thus $m-m^{\prime} \in \operatorname{Ker}\{a x+b y: M \rightarrow M\} \subseteq \Omega(M)$. It follows that $m$ is in $\Omega(M)$, and hence

$$
\operatorname{Ker}\{(a x+b y): \bar{M} \rightarrow \bar{M}\} \subset \Omega(M) / x \Omega(M) \text {. }
$$

The second assertion follows by dualization and an application of Proposition 7.8.

Theorem 8.6. If $M$ is a $k G$-module of constant rank, then $\mathfrak{I}(M)=x \Re(M)$.

Proof. Observe that $x \Re(M) \subseteq \mathfrak{I}(M)$, because $x \Re(M)=(a x+b y) \Re(M)$ for any $\langle a, b\rangle \in \mathbb{P}^{1}(k)$. To prove $\mathfrak{I}(M) \subseteq x \Re(M)$, we apply Lemma 8.5. Namely, Proposition 8.4 and Lemma 8.5 imply that

$$
\mathfrak{I}(M)=\left(\Omega\left(M^{\#}\right)\right)^{\perp} \subset\left((M / x \Omega(M))^{\#}\right)^{\perp}=x \Omega(M) .
$$

To formulate the next theorem, we find it convenient to introduce the dual notion of the equal images property of Definition 2.3. As before, if $\alpha_{K}: K[t] /\left(t^{p}\right) \rightarrow K G$, is a $\pi$-point, we let $\ell_{\alpha}=\alpha_{K}(t) \in K G$. 
Definition 8.7. A finite dimensional $k G$-module $M$ is said to have the equal kernels property if

$$
\left(\operatorname{Ker}\left\{\ell_{\alpha}: M_{K} \rightarrow M_{K}\right\}\right)_{\Omega}=\left(\operatorname{Ker}\left\{\ell_{\beta}: M_{L} \rightarrow M_{L}\right\}\right)_{\Omega}
$$

for any two $\pi$-points $\alpha_{K}: K[t] /\left(t^{p}\right) \rightarrow K G, \beta_{L}: L[t] /\left(t^{p}\right) \rightarrow L G$ and any field extension $\Omega$ of both $K, L$.

As an example, notice that if $M$ has constant rank, then $M / x \Re(M)$ has the equal kernels property by Lemma 8.5.

Remark 8.8. In Example 2.2, the module $M$ fails to have the equal kernels property, even though the kernel of any operator of the form $a x+b y$ on $M$ is independent of the choice of $\langle a, b\rangle \in \mathbb{P}^{1}(k)$. That is, a field extension is necessary in order to expose the failure of the property. A similar phenomenon occurs for the equal images property.

As in the case of the equal images property, one can prove analogs of Propositions 2.5 and 2.6 for the equal kernels property. Here, image must be exchanged for kernel and radical for socle. So, for example, for the equal kernels property, the equation at the end of Proposition 2.5 would read

$$
\operatorname{Ker}\left\{\sum_{i=1}^{r} a_{i} x_{i}: M_{K} \rightarrow M_{K}\right\}=\operatorname{Soc}\left(M_{K}\right) \text {. }
$$

The proofs can be constructed in a similar way. However, such results can also be verified using the following proposition.

Proposition 8.9. A $k G$-module $M$ has the equal kernels property if and only if $M^{\#}$ has the equal images property.

Proof. Suppose that $M$ has the equal kernels property. Let $K$ be an extension of $k$ and let $\alpha_{K}: K[t] /\left(t^{p}\right) \rightarrow K G$ be a $\pi$-point. Let $\ell=\alpha_{K}(t)$. Then, the dual statement of equation (23) says that

$$
\operatorname{Ker}\left\{\ell: M_{K} \rightarrow M_{K}\right\}=\left\{g: M_{K}^{\#} \rightarrow K \mid g \circ \ell^{\#}=0\right\}=\left(\ell M_{K}^{\#}\right)^{\perp} .
$$

Because $\operatorname{Ker}\left\{\ell: M_{K} \rightarrow M_{K}\right\}=\operatorname{Ker}\left\{x: M_{K} \rightarrow M_{K}\right\}$, we have that

$$
\ell M_{K}^{\#}=\left(\left(\ell M_{K}^{\#}\right)^{\perp}\right)^{\perp}=\left(\left(x M_{K}^{\#}\right)^{\perp}\right)^{\perp}=x M_{K}^{\#} .
$$

Hence $M^{\#}$ has the equal images property. The proof in the other direction is similar. 
If $M$ is a $k G$-module and $W \subset M$ is a submodule, then we denote by $x^{-1} W \subset M$ the $k G$-submodule consisting of elements $m \in M$ satisfying $x m \in W$.

We verify by inspection that for any submodule $W \subset M$ and any $i>0$,

$$
\left(\left(x^{i}\right)^{-1}(W)\right)^{\perp}=x^{i \#}\left(W^{\perp}\right) .
$$

Here, $x^{i \#}: M^{\#} \rightarrow M^{\#}$ sends $f$ to $f \circ x^{i}$, where $x^{i}: M \rightarrow M$ is the action of $x^{i} \in k G$ on $M$. With this notation, we can prove the following.

Theorem 8.10. Let $M$ be a module of constant rank and let $W \subset M$ be its generic kernel. Then we have an increasing filtration of $M$,

$$
x^{p-1}(W) \subseteq x^{p-2}(W) \subseteq \cdots \subseteq x(W) \subseteq W \subseteq x^{-1}(W) \subseteq \cdots \subseteq x^{1-p}(W) \subseteq M
$$

with the property that $x^{i}(W)$, for $i \geq 0$ has the equal images property and that $M / x^{j}(W)$ for $j<0$ has the equal kernels property. Moreover, for any $\ell=a x+b y$ with $\langle a, b\rangle \in \mathbb{P}^{1}(k)$ and for all $j$, we have that $x^{j} W=\ell^{j} W$. Here, $x^{j}(W)$ denotes $\left(x^{j}\right)^{-1}(W)$ for $j<0$.

Proof. First observe that all of the submodules in the theorem are stable under field extension. So there is no loss in generality by assuming that $k$ is algebraically closed. Each of the $x^{-j} W$ is a submodule of $M$ because $k G$ is commutative.

Each of the modules $x^{i}(W)$ with $i>0$ is a radical power of $W=\Omega(M)$ and thus has the equal images property by Proposition 2.8 .

Using Proposition 8.4, Theorem 8.6, and (25), we conclude that

$$
\left(\left(x^{i}\right)^{-1}(W)\right)^{\perp}=x^{i \#}\left(W^{\perp}\right)=x^{i \#}\left(\widetilde{I}\left(M^{\#}\right)\right)=\left(x^{i+1}\right)^{\#}\left(\Omega\left(M^{\#}\right)\right) .
$$

Hence, $\left(\left(x^{i}\right)^{-1}(W)\right)^{\perp}=\left(M / x^{-i} W\right)^{\#}$ has the equal images property. Thus, Proposition 8.9 implies that $M / x^{-i}(W)$ has the equal kernels property.

For $\ell=a x+b y \neq 0$, we know that $x^{j} W=\ell^{j} W$ for $j \geq 0$ by the fact that $W$ has the equal images property. For negative values of $j$ the result is a consequence of the equal kernels property on the quotients. That is, for $j \leq 0$ we have that $x^{j-1} W=$ $x^{-1}\left(x^{j} W\right)$ is precisely the inverse image under the quotient map $M \rightarrow M /\left(x^{j} M\right)$ of the kernel of $x$ on $M /\left(x^{j} M\right)$. Because $M /\left(x^{j} M\right)$ has the equal kernels property, the kernel of multiplication by $x$ is the same as the kernel of multiplication by $\ell$. Therefore, $x^{-1}\left(x^{j} W\right)=\ell^{-1}\left(x^{j} W\right)=\ell^{-1}\left(\ell^{j} W\right)=\ell^{j-1} W$ as asserted.

Question 8.11. In Theorem 8.10, we know that the submodules $\Omega(M)$, and $x^{i} \Omega(M)$ have constant Jordan type for $i \geq 0$. Assuming that $M$ has constant Jordan type, do the modules $x^{-i} \Omega(M)$ also have constant Jordan type? If yes, then the characterization of the cyclic modules of constant Jordan type becomes much easier. 


\section{9. $n^{\text {th }}$-power generic kernels}

In this section, we assume that the field $k$ is algebraically closed. (We leave to the reader the elementary modifications required to extend this discussion to more general fields. This assumption is not necessary.) We fix a choice of generators $x, y$ of $\operatorname{Rad}(k G)$, with $k G \simeq k[x, y] /\left(x^{p}, y^{p}\right)$.

Suppose that $n$ is an integer with $1 \leq n \leq p-1$. For a subset $S \subset \mathbb{P}^{1}(k)$, define the submodule

$$
{ }_{S}^{n} M=\sum_{\langle a, b\rangle \in S} \operatorname{Ker}\left\{(a x+b y)^{n}: M \rightarrow M\right\}
$$

Since both $x$ and $y$ commute with each $a x+b y$ for $\langle a, b\rangle \in S,{ }_{S} M$ is a $k G$ submodule of $M$. Clearly, for any cofinite subset $S^{\prime} \subset S$, and any non-negative integer $n$ we have that ${ }_{S^{\prime}}^{n} M \subset{ }_{S}^{n} M$.

Definition 9.1. We define the $n^{\text {th }}$-power generic kernel of $M$ to be

$$
\Re^{n}(M)=\bigcap_{S \subset \mathbb{P}^{1}(k) \text { cofinite }}{ }_{S}^{n} M .
$$

Example 9.2. For the free cyclic $k G$-module, $k G$, we have that

$$
\Omega^{n}(k G)=\operatorname{Rad}^{p-n}(k G)=x^{-n+1} \Re(k G)
$$

for any $1 \leq n<p$. The case $n=1$ is in Example 7.2. The same sort of argument works also in this case. That is, any $\ell=a x+b y$ has Jordan type $p[p]$ on $k G$ and hence the kernel of $\ell^{n}$ on $k G$ is in $\ell^{p-n} k G \subseteq \operatorname{Rad}^{p-n}(k G)$. So $\Re^{n}(k G) \subseteq$ $\operatorname{Rad}^{p-n}(k G)$. To get the reverse inclusion, we note that $\ell^{p-n}$ is in the kernel of multiplication by $\ell^{n}$. Hence, some infinite subset (cofinite in $\mathbb{P}^{1}(k)$ ) of elements of the form $\ell^{p-n}$ is in $\Re^{n}(k G)$. By a Vandermonde determinant argument, some finite subset of these will generate $\operatorname{Rad}^{p-n}(k G)$.

Note that $\Omega^{1}(M)=\Omega(M)$ for any $M$. The $n^{\text {th }}$-power generic kernel enjoys some of the same properties as the generic kernel. For example. for each $n$ there must exist some non-empty open set $S_{n} \subseteq \mathbb{P}^{1}(k)$ such that $\Omega^{n}(M)={ }_{S_{n}}^{n} M$. If we let $S=\bigcap_{n=1}^{p-1} S_{n}$, then $S$ has the property that $\Omega^{n}(M)={ }_{S}^{n} M$ for all $n$.

The following proposition provides a partial generalization of Example 9.2 to other $k G$-modules.

Proposition 9.3. Suppose that $M$ is a $k G$-module of constant rank, and assume that $p>2$. Then for any $n=2, \ldots, p-1$ we have that

$$
\Omega^{n}(M) \subseteq x^{-n+1} \Re(M) .
$$


Proof. We prove the case that $n=2$. The rest follows by similar arguments which we leave to the reader. Suppose that $S$ is a subset of $\mathbb{P}^{1}(k)$ such that

$$
\Omega^{2}(M)={ }_{S}^{2} M=\sum_{\langle a, b\rangle \in S} \operatorname{Ker}\left\{(a x+b y)^{2}: M \rightarrow M\right\}
$$

and

$$
\Re(M)={ }_{S} M .
$$

If $\langle a, b\rangle \in S$ and if $m \in \operatorname{Ker}\left\{(a x+b y)^{2}: M \rightarrow M\right\}$, then $(a x+b y)^{2} m=0$ and hence, $(a x+b y) m \in \Re(M)$. So, $m \in(a x+b y)^{-1} \Re(M)=x^{-1} \Re(M)$ by Theorem 8.10. It follows that $\Omega^{2}(M) \subseteq x^{-1} M$.

On the other hand, the equality of Example 9.2 is not valid for general $k G$-modules as seen in the following example.

Example 9.4. Assume that $p>3$. Let $M$ be the $k G$-module represented by the diagram

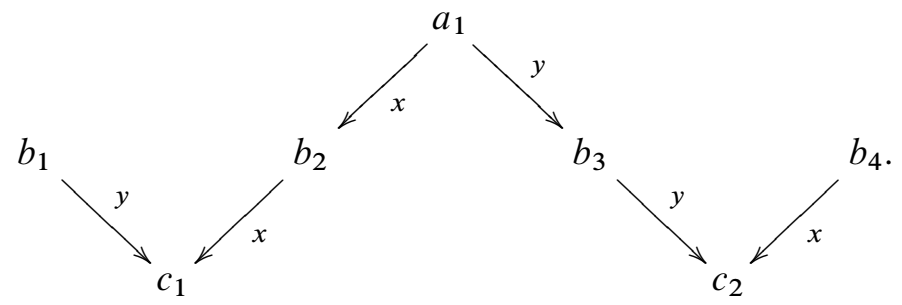

That is, $M$ has $k$-basis consisting of the elements $a_{1}, b_{1}, b_{2}, b_{3}, b_{4}, c_{1}, c_{2}$ and the matrices of $x$ and $y$ are given by

$$
x \rightarrow\left(\begin{array}{lllllll}
0 & 0 & 0 & 0 & 0 & 0 & 0 \\
0 & 0 & 0 & 0 & 0 & 0 & 0 \\
1 & 0 & 0 & 0 & 0 & 0 & 0 \\
0 & 0 & 0 & 0 & 0 & 0 & 0 \\
0 & 0 & 0 & 0 & 0 & 0 & 0 \\
0 & 0 & 1 & 0 & 0 & 0 & 0 \\
0 & 0 & 0 & 0 & 1 & 0 & 0
\end{array}\right), \quad y \rightarrow\left(\begin{array}{ccccccc}
0 & 0 & 0 & 0 & 0 & 0 & 0 \\
0 & 0 & 0 & 0 & 0 & 0 & 0 \\
0 & 0 & 0 & 0 & 0 & 0 & 0 \\
1 & 0 & 0 & 0 & 0 & 0 & 0 \\
0 & 0 & 0 & 0 & 0 & 0 & 0 \\
0 & 1 & 0 & 0 & 0 & 0 & 0 \\
0 & 0 & 0 & 1 & 0 & 0 & 0
\end{array}\right) .
$$

It is straightforward to see that $M$ has constant Jordan type [3] + [2] $+2[1]$. Note that for any $\langle a, b\rangle \in \mathbb{P}^{1}(k)$, we have that $(a x+b y)^{2} a_{1} \neq 0$ and that $(a x+$ by $)^{2} b_{j}=0$ for $j=1, \ldots, 4$. Consequently, $\Omega^{2}(M)$ is the submodule $N$ spanned by $b_{1}, \ldots, b_{4}, c_{1}, c_{2}$. Because $N$ is the direct sum of two $W$ modules, it is equal to its own generic kernel and is also the generic kernel of $M$. Therefore we have that

$$
\Omega^{2}(M)=N=\Omega(M) \neq x^{-1} \Re(M) .
$$


Motivated by Question 8.11, we ask the following.

Question 9.5. If $M$ has constant Jordan type, then does $\Omega^{n}(M)$ also have constant Jordan type?

\section{Some preliminaries on cyclic modules}

The purpose of this section is to develop some technical results that are essential for the proofs of the next section. The subject matter is something of a digression from the primary issues of the paper. The reader may wish to continue with the next section and return to this material only as needed.

Definition 10.1. Let $p_{1}(t), \ldots, p_{r}(t) \in k[t]$ be polynomials. For any $i>0$, we denote by $p_{j}^{(i)}(t)$ the $i^{\text {th }}$ derivative of $p_{j}(t)$. The Wronskian $W\left(p_{1}, \ldots, p_{r}\right)$ of $\left\{p_{1}(t), \ldots, p_{r}(t)\right\}$ is the following determinant:

$$
W\left(p_{1}, \ldots, p_{r}\right)=\operatorname{Det}\left(\begin{array}{cccc}
p_{1}(t) & p_{2}(t) & \cdots & p_{r}(t) \\
p_{1}^{(1)}(t) & p_{2}^{(1)}(t) & \cdots & p_{r}^{(1)}(t) \\
\cdots & \cdots & \cdots & \cdots \\
p_{1}^{(r-1)}(t) & p_{2}^{(r-1)}(t) & \cdots & p_{r}^{(r-1)}(t)
\end{array}\right)
$$

When the field $k$ has characteristic 0 , it is well known (cf. [12], pp. 524-525) that $W\left(p_{1}, \ldots, p_{r}\right)=0$ if and only if $p_{1}(t), \ldots, p_{r}(t)$ are linearly dependent. Moreover, if one of the polynomials $p_{i}(t)$ has degree at least $r$, then $W\left(p_{1}, \ldots, p_{r}\right)$ is not a constant polynomial. This is because $t$ would divide the leading term in every entry of the $i^{\text {th }}$ column. For a field of positive characteristic, we have the following.

Proposition 10.2. Let $k$ be a field of characteristic $p>0$ and consider polynomials $p_{1}(t), \ldots, p_{r}(t) \in k[t]$. Then $W\left(p_{1}, \ldots, p_{r}\right)=0$ if and only if $p_{1}(t), \ldots, p_{r}(t)$ are linearly dependent over $k\left(t^{p}\right) \subset k(t)$.

Proof. We first assume that $p_{1}(t), \ldots, p_{r}(t)$ are linearly dependent over $k\left(t^{p}\right)$ so that we have a non-trivial relation

$$
0=\sum_{i=1}^{r} e_{i} \cdot p_{i}(t) \quad \text { for } e_{i} \in k\left(t^{p}\right) .
$$

By taking derivatives, and using the fact that the derivative of every $e_{i}$ is zero, we 
obtain a system of equations

$$
\begin{aligned}
e_{1} p_{1}(t)+e_{2} p_{2}(t)+\ldots+e_{r} p_{r}(t) & =0, \\
e_{1} p_{1}^{(1)}(t)+e_{2} p_{2}^{(1)}(t)+\ldots+e_{r} p_{r}^{(1)}(t) & =0, \\
& \vdots \\
e_{1} p_{1}^{(r-1)}(t)+e_{2} p_{2}^{(r-1)}(t)+\ldots+e_{r} p_{r}^{(r-1)}(t) & =0 .
\end{aligned}
$$

This implies that the columns $\underline{C}_{1}, \ldots, \underline{C} r$ of the matrix

$$
\left(\begin{array}{cccc}
p_{1}(t) & p_{2}(t) & \cdots & p_{r}(t) \\
p_{1}^{(1)}(t) & p_{2}^{(1)}(t) & \cdots & p_{r}^{(1)}(t) \\
\cdots & \cdots & \cdots & \cdots \\
p_{1}^{(r-1)}(t) & p_{2}^{(r-1)}(t) & \cdots & p_{r}^{(r-1)}(t)
\end{array}\right)
$$

are linearly dependent over $k\left(t^{p}\right)$, and thus that the determinant of (28), which is $W\left(p_{1}, \ldots, p_{n}\right)$, is 0 .

Conversely, assume that $W\left(p_{1}, \ldots, p_{r}\right)=0$. We proceed by induction on $r$, the case $r=1$ being clear. So assume that $r>1$ and that $W\left(p_{1}, \ldots, p_{r-1}\right) \neq 0$. Without loss of generality, we may further assume that the right-most column $\underline{C}_{r}$ of (28) is a linear combination of the first $r-1$ columns $\underline{C}_{1}, \ldots, \underline{C}_{r-1}$. Hence we get a system of equations:

$$
\underline{C}_{r}=\lambda_{1} \underline{C}_{1}+\cdots+\lambda_{n-1} \underline{C}_{r-1}, \quad \lambda_{i} \in k(t) .
$$

Subtracting the first derivative of the $i^{\text {th }}$ equation (row) of (29) from the $(i+1)^{s t}$-row for $i=1, \ldots, r-2$, we conclude, using the product rule for derivatives, that

$$
\lambda_{1}^{\prime} p_{1}^{(i)}(t)+\cdots+\lambda_{r-1}^{\prime} p_{r-1}^{(i)}(t)=0, \quad 0 \leq i \leq r-2,
$$

where $\lambda_{i}^{\prime}$ is the derivative of $\lambda_{i}$. Since $W\left(p_{1}, \ldots, p_{r-1}\right) \neq 0$, we see that $\lambda_{i}^{\prime}=0$, for all $1 \leq i \leq r-1$. Thus, the first row of (29) gives a linear dependence over $k\left(t^{p}\right)$ of $p_{1}, \ldots, p_{r}$.

Corollary 10.3. With notation as in Proposition 10.2, assume that the degree as a function of $t$ of each $p_{i}(t)$ is less than $p$. Then $W\left(p_{1}, \ldots, p_{r}\right) \neq 0$ if and only if $p_{1}(t), \ldots, p_{r}(t)$ are linearly independent over $k$. Moreover, if $\operatorname{deg}\left(p_{i}(t)\right) \geq r$ for some $i$, then $W\left(p_{1}(t), \ldots, p_{r}(t)\right)$ is not constant as a function of $t$.

Proof. If $W\left(p_{1}, \ldots, p_{r}\right) \neq 0$, then Proposition 10.2 asserts that $p_{1}(t), \ldots, p_{r}(t)$ are linearly independent over $k\left(t^{p}\right)$ and thus also linearly independent over $k$.

Conversely, assume that $p_{1}(t), \ldots, p_{r}(t)$ are linearly independent over $k$. Since each $p_{i}(t)$ has degree less than $p$, we conclude that $r \leq p$. Using elementary 
transformations with constant coefficients on the system $\left\{p_{1}(t), \ldots, p_{r}(t)\right\}$, which do not change the value of the Wronskian $W\left(p_{1}, \ldots, p_{r}\right)$, we may assume that $\operatorname{deg}\left(p_{i}(t)\right) \neq \operatorname{deg}\left(p_{j}(t)\right)$ for $i \neq j$. That is, if $\operatorname{deg}\left(p_{i}(t)\right)=\operatorname{deg}\left(p_{j}(t)\right)$, then there is a scalar $c$ such that $q_{j}(t)=p_{j}(t)-c p_{i}(t)$ has lower degree. Replacing $p_{j}(t)$ by $q_{j}(t)$ amounts to an elementary column operation which does not change the value of the Wronskian. As a result, we may assume that $p_{1}(t), \ldots, p_{r}(t)$ all have different degrees. Hence, they are linearly independent over $k\left(t^{p}\right)$. By Proposition 10.2, the Wronskian is not zero.

To prove the final statement of the corollary, we note that by the above argument we may assume that no two of the polynomials $p_{1}(t), \ldots, p_{r}(t)$ have the same degree. Let $q_{i}(t)$ denote the leading term of $p_{i}(t)$. It is not difficult to see that the leading term of $W\left(q_{1}(t), \ldots, q_{r}(t)\right)$ is the leading term of $W\left(p_{1}(t), \ldots, p_{r}(t)\right)$. Because the degree of the elements $q_{i}(t)$ are different and less than $p$, they are independent over $k\left(t^{p}\right)$ and the Wronskian is not zero. If for some $i$ the degree of $q_{i}(t)$ is at least $r$, then every entry in the $i^{\text {th }}$ column of matrix of the Wronskian $W\left(q_{1}(t), \ldots, q_{r}(t)\right)$ is divisible by $t$. It follows that the leading term of $W\left(p_{1}(t), \ldots, p_{r}(t)\right)$ is divisible by $t$ and hence it is not constant.

Proposition 10.4. Let $k$ be an algebraically closed field of characteristic $p \geq 0$. Let $k[t]_{\leq n}$ denote the $k$-vector space of polynomials of degree at most $n$ for some $n<p$. Assume that $n<p$ if the characteristic $p$ is positive. Fix an integer $j<n$, and suppose that $V \subset k[t]_{\leq n}$ is a proper subspace having the properties that

(i) V contains a polynomial of degree $j+1$, and

(ii) for any $a \in k$, there exists some $f(t) \in V$ such that $(t-a)^{j}$ divides $f(t)$ (i.e., such that $a$ is a root of multiplicity at least $j$ of $f(t))$.

Then there exist some $a \in k$ and some $g(t) \in V$ such that $(t-a)^{j+1}$ divides $g(t)$.

Proof. Observe that $\operatorname{Dim}\left(k[t]_{\leq n}\right)=n+1$. Let $r$ denote $\operatorname{Dim}(V)$. Choose a basis $p_{1}(t), p_{2}(t), \ldots, p_{r}(t)$ of $V$ with the property that

$$
d_{1}=\operatorname{deg}\left(p_{1}(t)\right)>d_{2}=\operatorname{deg}\left(p_{2}(t)\right)>\cdots>d_{r}=\operatorname{deg}\left(p_{r}(t)\right) .
$$

To emphasize its dependence on $t$, we let $D(t)=W\left(p_{1}(t), \ldots, p_{r}(t)\right)$ :

$$
D(t)=\operatorname{Det}\left(\begin{array}{cccc}
p_{1}(t) & p_{2}(t) & \cdots & p_{r}(t) \\
p_{1}^{(1)}(t) & p_{2}^{(1)}(t) & \cdots & p_{r}^{(1)}(t) \\
\cdots & \cdots & \cdots & \cdots \\
p_{1}^{(r-1)}(t) & p_{2}^{(r-1)}(t) & \cdots & p_{r}^{(r-1)}(t)
\end{array}\right)
$$

be the Wronskian. By Corollary 10.3 (in the case that $p>0$ ), and Condition (i), $D(t)$ is not a constant polynomial. In particular, it is not zero. 
We first verify that $\operatorname{Dim} V=r \geq j+1$. By Condition (ii) for any element $a \in k$ there exists a non-zero polynomial $f(t)=\sum_{i=1}^{r} \mu_{i} p_{i}(t)$ in $V$ such that $f(t)$ is divisible by $(t-a)^{j}$. Consider the system of equations given by the equality

$$
\left(\begin{array}{c}
f(t) \\
f^{(1)}(t) \\
\cdots \\
f^{(r-1)}(t)
\end{array}\right)=\left(\begin{array}{cccc}
p_{1}(t) & p_{2}(t) & \cdots & p_{r}(t) \\
p_{1}^{(1)}(t) & p_{2}^{(1)}(t) & \cdots & p_{r}^{(1)}(t) \\
\cdots & \cdots & \cdots & \cdots \\
p_{1}^{(r-1)}(t) & p_{2}^{(r-1)}(t) & \cdots & p_{r}^{(r-1)}(t)
\end{array}\right)\left(\begin{array}{c}
\mu_{1} \\
\mu_{2} \\
\cdots \\
\mu_{r}
\end{array}\right) .
$$

If $r<j+1$, then the column on the left of (31) vanishes when evaluated at $t=a$, so the fact that $f(t)$ is not zero implies that $D(a)=0$ (i.e., $a$ is a root of $D(t)$ ). As this happens for any $a$ in $k$ we have a contradiction to the fact that $D(t)$ can have only finitely many roots. Hence, we conclude that $r \geq j+1$.

We now proceed to show that some element of $V$ has a root $a$ of multiplicity $j+1$. This would prove the proposition. Suppose to the contrary that there is even a single element $\gamma \in k$ such that $V \cap U=\{0\}$, where $U$ is the space of all multiples of $(t-\gamma)^{j+1}$ in $k[t]_{\leq n}$. Then the dimension of $V$ is at most equal to the dimension of $k[t]_{\leq n}$ minus the dimension of $U$. That is,

$$
r=\operatorname{Dim}(V) \leq(n+1)-(n+1-(j+1))=j+1 .
$$

Therefore, for the rest of the proof, we may assume that $r=j+1$.

Because the Wronskian $D(t)$ is not a constant polynomial and the field $k$ is algebraically closed, $D(t)$ must have a root $a \in k$. Then the determinant of the matrix

$$
\left(\begin{array}{cccc}
p_{1}(a) & p_{2}(a) & \cdots & p_{r}(a) \\
p_{1}^{(1)}(a) & p_{2}^{(1)}(a) & \cdots & p_{r}^{(1)}(a) \\
\cdots & \cdots & \cdots & \cdots \\
p_{1}^{(r-1)}(a) & p_{2}^{(r-1)}(a) & \cdots & p_{r}^{(r-1)}(a)
\end{array}\right)
$$

is zero, and we must have that its columns are linearly dependent vectors in $k^{r}$. Suppose that $\mu_{1}, \ldots, \mu_{r}$ are the coefficients of a non-trivial dependence relation among the columns. Then we have that if $f(t)=\sum_{i=1}^{r} \mu_{i} p_{i}(t)$, then $f(a)=0$ and moreover, $f^{(i)}(a)=0$ for all $i=1, \ldots, r-1$. Therefore $f(t) \in V$ and $a$ is a root of $f(t)$ of multiplicity $r$. This proves the proposition.

Corollary 10.5. Let $k$ be a field of characteristic $p \geq 0$. Let $k[x, y]_{n}$ denote the space of homogeneous polynomials of degree $n$ in the variables $x$ and $y$. Assume that $n<p$ if $p>0$. Fix an integer $j<n$. Suppose that $V$ is a subspace of $k[x, y]_{n}$ having the following properties.

(i) The space $V$ contains an element which is not divisible by $y^{n-j-1}$.

(ii) For any $a \in k$, there exists an element $f(x, y)$ in $V$ such that $f(x, y)$ is divisible by $(x-a y)^{j}$.

Then for some $a \in k, V$ contains an element $f(x, y)$ which is divisible by $(x-a y)^{j+1}$. 
Proof. Let $\psi: k[x, y]_{n} \rightarrow k[t]_{\leq n}$ be the linear transformation of vector spaces obtained by sending $x$ to $t$ and $y$ to 1 . Then under the isomorphism $\psi$, conditions (i) and (ii) of the corollary translate into conditions (i) and (ii) of Proposition 10.4. Moreover, the conclusion of Proposition 10.4 translates to the conclusion of the corollary.

\section{Cyclic modules of constant Jordan type}

Our objective in this section is to show that any cyclic module of constant Jordan type is a quotient of the algebra by a power of the augmentation ideal of the algebra. We break up the argument into two cases. For the first case, Theorem 11.1 completely settles the case of a field of characteristic 0 and goes a part of the way to verifying this result for fields of positive characteristic.

Theorem 11.1. Let $k$ be a field of any characteristic. Consider a cyclic $k[x, y]$ module $M$ which is finite dimensional and has constant Jordan type. Assume that $x$ and $y$ act as nilpotent operators on $M$. Let $m \in M$ be a generator and let $n$ be the least integer such that $x^{n+1} m=0$. So $x^{n} \cdot m \neq 0$. If $k$ has positive characteristic $p$, then assume that $n<p$. Then

$$
M \simeq k[x, y] / I^{n+1}
$$

where $I=(x, y) \subset k[x, y]$ denotes the augmentation ideal.

Proof. Observe that the hypothesis that $M$ has constant Jordan type implies that $(x+a y)^{n+1} m=0$ for all $a$ in $k$. Also we may assume that $n>0$ since otherwise $M \simeq k$. Hence, all monomials in $x, y$ of degree $n+1$ kill $M$, so that $M$ is a quotient of $k[x, y] / I^{n+1}$. Note here that even in the case that $k$ is the prime field with $p$ elements, the assumption that $n<p$ assures us that the elements $(x+a y)^{n+1}$ span the space of all homogeneous polynomials of degree $n+1$ in $k[x, y]$.

Without loss of generality, we may assume that the field $k$ is algebraically closed. That is, suppose that $K$ is the algebraic closure of $k$. Because $M$ has constant Jordan type, so also does $K \otimes_{k} M$. Also, since $K \otimes_{k}-$ is an exact functor, a proof that $K \otimes_{k} M \simeq K[x, y] /\left(I^{\prime}\right)^{n+1}$ implies that $M \simeq k[x, y] / I^{n+1}$ where $I^{\prime}=K \otimes I$ is the augmentation ideal of $K[x, y]$. Hence we assume that $k=K$.

Our strategy is to show that the Jordan type of $M$ has $n+1$ blocks, one of size $j$ for each $1 \leq j \leq n+1$, thereby showing that the induced map $k[x, y] / I^{n+1} \rightarrow M$ is an isomorphism. Note that there is exactly one block of size $j=n+1$, since the number of blocks of $M$ of size $n+1$ equals $\operatorname{Dim}\left(x^{n} \cdot M\right)$. Yet, $x^{n} \cdot M=\left\langle x^{n} m\right\rangle$, and hence has dimension 1.

We proceed by downward induction on $j$. We assume that the Jordan type of $M$ has exactly 1 block each of size $n+1, \ldots, j+1$. In other words, we assume that 
elements in the set

$$
\begin{aligned}
& \left\{m, \ldots, x^{n} m, y m, x y m, \ldots, x^{n-1} y m, \ldots, y^{n-j} m, \ldots, x^{j} y^{n-j} m\right\} \\
& =\left\{x^{e} y^{f} m \mid 0 \leq e+f \leq n, f \leq n-j\right\}
\end{aligned}
$$

are linearly independent. This condition is equivalent to the condition that the elements in the set

$$
S=\left\{x^{n} m, x^{n-1} y m, \ldots, x^{j} y^{n-j} m\right\}=\left\{x^{e} y^{f} m \mid e+f=n, f \leq n-j\right\}
$$

are linearly independent. So $m$ is not annihilated by any homogeneous polynomial of degree $n$ divisible by $x^{j}$. Because $M$ has constant Jordan type, $m$ is not annihilated by any homogeneous polynomial divisible by $(x-a y)^{j}$ for any $a$ in $k$.

Suppose that $M$ does not have a block of size $j$. Then $x^{j-1} y^{n-j+1} m$ is a linear combination of the elements in $S$. That is, $m$ is annihilated by some homogeneous polynomial of degree $n$ divisible by $x^{j-1}$. We may repeat verbatim this discussion with $x$ replaced by $x-a y$ for any $a \in k$. We conclude that $m$ is annihilated by some homogeneous polynomial of degree $n$ divisible by $(x-a y)^{j-1}$ for any $a \in k$.

Let $V$ denote the vector space of all homogeneous polynomials $f(x, y)$ of degree $n$ which annihilate $m$. By the above, we have that $V$ satisfies both conditions (i) and (ii) of Corollary 10.5. Consequently, that corollary tells us that $V$ contains a polynomial $f(x, y)$ which is divisible by $(x-a y)^{j}$ for some $a \in k$. But this is a contradiction. So there must also be a block of size $j$.

As we see in Corollary 11.3, the following theorem together with Theorem 11.1 easily implies the identification of all cyclic $k G$-modules of constant Jordan type.

Theorem 11.2. Let $k$ be a field of characteristic $p>0$. Assume that $M$ is a cyclic $k[x, y] /\left(x^{p}, y^{p}\right)$-module of constant Jordan type such that $x^{p-1} \cdot M \neq 0$. Set $s$ equal to the number of Jordan blocks of $M$ of size $p$. Then $s>0$ and

$$
M \simeq A / I^{p+s-1}
$$

where $I=(x, y)$ is the augmentation ideal of $A$.

Proof. As in the proof of the last theorem, we can assume that the field $k$ is algebraically closed. Let $A_{x}=k[x] /\left(x^{p}\right) \subset A$. The (constant) Jordan type of $M$ is the type of $M$ as an $A_{x}$-module. We readily verify that $A / I^{p+s-1}$ has Jordan type $s[p]+[p-1]+\cdots+[s]$.

The number of blocks of size $p$ of $M$ as an $A_{x}$-module equals the dimension of $N=x^{p-1} M$. Note that $N$ is a module over $A_{y}$ of dimension $s$ and is generated by $x^{p-1} m$. Consequently, $y^{s} N=0$, and we must have also that $x^{p-1} y^{s} m=0$. 
Because $M$ has constant Jordan type, we conclude that $(x-\gamma y)^{p-1} y^{t} m=0$ for any $\gamma \in k$, any $t \geq s$. This implies that

$$
x^{i} y^{j} m=0, \quad i+j \geq p-1+s .
$$

Hence, $A \rightarrow M$ factors through the surjection $A \rightarrow A / I^{p+s-1}$. In particular, to prove the theorem it suffices to prove that the Jordan type of $M$ is the same as that of $A / I^{p+s-1}$ as an $A_{x}$-module.

We proceed as follows. Suppose that the Jordan type of $M$ includes $s$ blocks of length $p$ and also exactly one block of each size $p-1, p-2, \ldots, p-j+1$. That is suppose that $p-j$ is the largest integer such that there is no block of size $p-j$ for $p-j \geq s$. As an $A_{x}$-module, the blocks of size $p$ can be assumed to be generated by $m, y m, \ldots, y^{s-1} m$, as discussed before. The block of size $s-i$ is generated by $y^{s-1+i} m$, for $i=1, \ldots, j-1$. Hence the sum of the $A_{x}$-socles of these blocks is spanned by the set of elements $S=S_{1} \cup S_{2}$ where

$$
S_{1}=\left\{x^{p-1} m, x^{p-1} y m, \ldots, x^{p-1} y^{s-2} m\right\}
$$

and

$$
S_{2}=\left\{x^{p-1} y^{s-1} m, \ldots, x^{p-j} y^{s+j-2} m\right\} .
$$

Because these elements lie in different blocks of $M$ as an $A_{x}$-module, they must be $k$-linearly independent. Moreover, each is annihilated by multiplication by $x$. Because there is no block of size $p-j$ we must have that there exist scalars $a_{0}, \ldots, a_{s-2}, b_{1}, \ldots, b_{j}$ such that

$$
x^{p-j-1} y^{s+j-1} m=\sum_{i=0}^{s-2} a_{i} x^{p-1} y^{i}+\sum_{i=1}^{j} b_{i} x^{p-i} y^{s+i-2} .
$$

Multiplying by $y$, which annihilates all of the terms of degree $p+s-2$, we get that

$$
a_{0} x^{p-1} y m+a_{1} x^{p-1} y^{2} m+\cdots+a_{s-2} x^{p-1} y^{s-1} m=0
$$

Hence the linear independence of the elements in the set $S$ implies that $a_{i}=0$ for all $i$. Consequently by equation (35), there exists a homogeneous polynomial $f(x, y)$ of degree $p-1$ in $k[x, y]$ with the properties that

$$
f(x, y) y^{s-1} m=0,
$$

and that $f(x, y)$ is divisible by $x^{p-j-1}$.

Now let $V$ be the $k$-space of all those elements $f(x, y)$ in $k[x, y]_{p-1}$ for which $f(x, y) y^{s-1} m=0$. We have shown that $V$ contains an element which is divisible by $x^{p-j-1}$. We can repeat the same argument with $x$ replaced by $x-a y$ for any $a \in k$. 
Hence, $V$ must contain an element which is divisible by $(x-a y)^{p-j-1}$ for any $a$ in $k$. This says that $V$ satisfies condition (ii) of the hypothesis of Corollary 10.5.

Next we want to check that condition (i) of the hypothesis of Corollary 10.5 is satisfied at least for some choice of the variables. Suppose not. Then we must have that $V$ contains every polynomial of the form $(x-a y)^{p-j-1} y^{j}$ for every $a \in k$. That is, for every $a \in k, V$ must contain a non-zero polynomial which is divisible by both $(x-a y)^{p-j-1}$ and $y^{j}$. Because the elements are relatively prime, that polynomial must be a scalar multiple of the product of the two. Recalling the definition of the space $V$, we see that for every $a \in k$ we have that

$$
(x-a y)^{p-j-1} y^{s+j-1} m=0 .
$$

Because $M$ has constant Jordan type we can make linear changes in the variables and repeat all of the same arguments. That is we can let $\bar{x}=a x+b y$ and $\bar{y}=c x+d y$, as long as the vectors $(a, b)$ and $(c, d)$ are linearly independent in $k^{2}$. If condition (i) fails for $\bar{x}$ and $\bar{y}$, then we have that

$$
(a x+b y)^{p-j-1}(c x+d y)^{s+j-1} m=0
$$

Now the elements $(a x+b y)^{p-j-1}(c x+d y)^{s+j-1} \operatorname{span} I^{p-s-2}$. Hence if condition (i) fails for all such choices of $\bar{x}=a x+b y$ and $\bar{y}=c x+d y$ we must have that $I^{p-s-2} M=\{0\}$ which contradicts our hypothesis that there are $s$ blocks of size $p$ in the Jordan type of $M$.

From all of the above, we may assume that conditions (i) and (ii) of Corollary 10.5 are satisfied. Hence the corollary implies that some element $f(x, y)$ of $V$ is divisible by $(x-a y)^{p-j}$ for some $a \in k$. Indeed, making the change of variables, replacing $x$ - ay by $x$, we may assume that there is a non-zero polynomial $g(x, y)$ of degree $j-1$ such that $g(x, y) x^{p-j-1}$ is contained in $V$. Hence,

$$
g(x, y) y^{s-1} x^{p-1} m=0 .
$$

This relation contradicts the linear independence of the set $S$, and hence, proves the theorem.

Corollary 11.3. Let $k$ be a field of characteristic $p>0$. Suppose that $A=$ $k[x, y] /\left(x^{p}, y^{p}\right)$ is the group algebra of an elementary abelian p-group of rank 2. If $M=A \cdot m$ is a cyclic A-module having constant Jordan type, then $M \cong A / I^{t}$ for some $t$, where $I$ is the augmentation ideal of $A$.

Proof. If $x^{p-1} m=0$, then we may regard $M$ simply as a $k[x, y]$-module and invoke Theorem 11.1 to prove the result. Otherwise we apply Theorem 11.2. 


\section{Further determination of bundles}

In this final section, we consider the bundles on $\mathbb{P}^{1}$ associated to certain $k G$-modules which we have considered. We use the notation of Section 6.

Proposition 12.1. Suppose that $M$ is a module of constant rank. Then we have that $\Omega(M)={ }_{\mathbb{P}^{1}(k)} M$. That is, if $\ell=a x+$ by with $(a, b) \neq(0,0)$ then $\operatorname{Ker}\{\ell: M \rightarrow$ $M\} \subseteq \Re(M)$. Consequently, if $M$ is a module of constant rank, then

$$
\operatorname{Ker}\{\ell: M \rightarrow M\}=\operatorname{Ker}\{\ell: \Re(M) \rightarrow \Re(M)\} .
$$

Proof. The proof of the first assertion is a straightforward application of Proposition 7.6. The second assertion follows from the first, because one always has the inclusion $\operatorname{Ker}\{\ell: \Omega(M) \rightarrow \Omega(M)\} \subset \operatorname{Ker}\{\ell: M \rightarrow M\}$.

Proposition 12.1 has the following "geometric formulation", for the operator $\widetilde{\Theta}_{G}$ on $\mathcal{O} \otimes M$ (respectively, $\mathcal{O} \otimes \Omega(M)$ ) has kernel above $\ell \in \mathbb{P}^{1}(k)$ given by $\operatorname{Ker}\{\ell: M \rightarrow M\}$ (resp., $\operatorname{Ker}\{\ell: \Omega(M) \rightarrow \Omega(M)\}$ ).

Proposition 12.2. Suppose that $M$ is a module of constant rank. Then we have a coincidence of kernel bundles:

$$
\operatorname{Ker}\left\{\widetilde{\Theta}_{G}, \mathcal{O} \otimes M\right\}=\operatorname{Ker}\left\{\widetilde{\Theta}_{G}, \mathcal{O} \otimes \Omega(M)\right\} .
$$

We apply Proposition 12.2 to compute explicitly the kernel bundles for all cyclic $k G$-modules of constant Jordan type.

Proposition 12.3. Let $M$ be a cyclic $k G$-module of constant Jordan type, so that $M \simeq k G / I^{t}$ for some $1 \leq t \leq 2 p$.

(1) If $t \leq p$, then $\operatorname{Ker}\left\{\widetilde{\Theta}_{G}, \mathcal{O} \otimes M\right\}$ is a free $\mathcal{O}$-module of rank $t$.

(2) If $t>p$, then $\Re(M)=\Omega\left(W_{p, t-p+1}\right)$, so that

$$
\left.\operatorname{Ker}\left\{\widetilde{\Theta}_{G}, \mathcal{O} \otimes M\right\} \simeq \operatorname{Ker}\left\{\widetilde{\Theta}_{G}, \mathcal{O} \otimes W_{t-p+1, p}\right)\right\}
$$

is explicitly determined by Proposition 6.4 .

Proof. If $t \leq p$, then the kernel of the endomorphism $\alpha_{(a, b)}(u)$ on $M$ is the socle of $M$ for any $\alpha_{a, b}: k[u] /\left(u^{p}\right) \rightarrow k G$ with $(0,0) \neq(a, b)$. This immediately implies part (1).

By Proposition 12.2 and the fact that $W_{p, t-p+1}$ equals its own generic kernel, in order to prove part (3) it suffices to verify the equality $\Re(M)=\Re\left(W_{p, t-p+1}\right)$. This is easily done by inspection. 
The generic kernels for the modules $\Omega^{n}(k)$ are calculated in Example 7.12. This information is used in the proof of the following.

Proposition 12.4. Suppose that $n>1$, then there are short exact sequences of vector bundles

$$
\begin{aligned}
& 0 \longrightarrow \operatorname{Ker}\left\{\tilde{\Theta}_{G}, \mathcal{O} \otimes W_{p-1, p-1}\right\}^{\oplus n} \longrightarrow \operatorname{Ker}\left\{\tilde{\Theta}_{G}, \mathcal{O} \otimes \Omega^{2 n-1}(k)\right\} \\
& \longrightarrow \mathcal{O}(-n p+1) \longrightarrow 0
\end{aligned}
$$

and

$$
\begin{aligned}
0 \longrightarrow & \operatorname{Ker}\left\{\widetilde{\Theta}_{G}, \mathcal{O} \otimes W_{p, p-1}\right\}^{\oplus n} \longrightarrow \operatorname{Ker}\left\{\widetilde{\Theta}_{G}, \mathcal{O} \otimes \Omega^{2 n}(k)\right\} \\
& \longrightarrow \mathcal{O}(-n p) \longrightarrow 0 .
\end{aligned}
$$

If $\zeta \in \mathrm{H}^{n}(G, k)$ is nilpotent, then the module $L_{\zeta}$ has constant rank, though not constant Jordan type. In this case we calculate that

$$
\operatorname{Ker}\left\{\widetilde{\Theta}_{G}, \mathcal{O} \otimes L_{\zeta}\right\} \simeq \operatorname{Ker}\left\{\widetilde{\Theta}_{G}, \mathcal{O} \otimes \Omega^{2 n}(k)\right\} .
$$

Proof. From Example 7.12, $\Omega\left(\Omega^{2 n-1}(k)\right) / \operatorname{Rad}^{2}\left(\Omega\left(\Omega^{2 n-1}(k)\right)\right) \cong W_{n p, 2}$. Thus, the kernel of $\widetilde{\Theta}_{G}$ on the top (head) of $\mathcal{O} \otimes \Omega\left(\Omega^{2 n-1}(k)\right)$ is isomorphic to $\mathcal{O}(-n p+1)$ exactly as in Example 6.3. Consequently, by Proposition 12.2 we need only compute the kernel of $\widetilde{\Theta}_{G}$ on $\mathcal{O} \otimes \operatorname{Rad}\left(\Omega\left(\Omega^{2 n-1}(k)\right)\right)$. However, an easy computation reveals that $\operatorname{Rad}\left(\Omega\left(\Omega^{2 n-1}(k)\right)\right)$ is isomorphic to a direct sum of $n$ copies of $W_{p-1, p-1}$. This verifies the exactness of the first sequence. The verification of the second is similar.

We calculate that $\Omega\left(\Omega^{2 n}(k)\right) / \operatorname{Rad}\left(\Omega\left(\Omega^{2 n}(k)\right)\right) \cong W_{n p+1,2}$ and hence, the contribution of the top of $\left.\Omega\left(\Omega^{2 n}(k)\right)\right)$ to the kernel of $\widetilde{\Theta}_{G}$ is a copy of the bundle $\mathcal{O}(-n p)$ as in Example 6.3. Finally, we observe that $\operatorname{Rad}\left(\Omega\left(\Omega^{2 n}(k)\right)\right)$ is isomorphic to a direct sum of $n$ copies of $W_{p, p-1}$ and we are done.

The last statement follows also from the calculation of Example 7.13.

\section{References}

[1] J. Carlson and E. Friedlander, Exact category of modules of constant Jordan type. In Algebra, arithmetic, and geometry: in honor of Yu. I. Manin. Vol. I, Progr. Math. 269, Birkhäuser, Boston, MA, 2009, 267-290. Zbl 05790985 MR 2641174 615, 621, 623

[2] J. Carlson, E. Friedlander, and J. Pevtsova, Modules of constant Jordan type. J. Reine Angew. Math. 614 (2008), 191-234. Zbl 1144.20025 MR 2376286 609, 618

[3] J. Carlson, L. Townsley, L. Valero-Elizondo, and M. Zhang, Cohomology rings of finite groups. Algebras Appl. 3, Kluwer Academic Publishers, Dordrecht 2003. Zbl 1056.20039 MR 2028960 615, 638 
[4] V. Dlab and K. Ringel, Indecomposable representations of graphs and algebras. Mem. Amer. Math. Soc. 6 (1976), no. 173. Zbl 0332.16015 MR 0447344629

[5] E. Friedlander and J. Pevtsova, П-supports for modules for finite group schemes. Duke Math. J. 139 (2007), 317-368 Zbl 1128.20031 MR 2352134612

[6] E. Friedlander and J. Pevtsova, Constructions for infinitesimal group schemes. Trans Amer. Math. Soc., to appear; Preprint 2010. arXiv:0802.2918v2 [math.RT] 610, 629, 630, 631

[7] E. Friedlander, J. Pevtsova, and A. Suslin, Generic and maximal Jordan types. Invent. Math. 168 (2007), 485-522. Zbl 1117.14047 MR 2299560 609, 610, 614, 636

[8] A. Grothendieck, Sur la classification des fibrés holomorphes sur la sphère de Riemann. Amer. J. Math. 79 (1957), 121-138. Zbl 0079.17001 MR 0087176613

[9] R. Hartshorne, Algebraic geometry. Grad. Texts in Math. 52, Springer-Verlag, Berlin 1977. Zbl 0367.14001 MR 0463157 613, 631

[10] K. Hoffman and R. Kunze, Linear Algebra. Second edition, Prentice-Hall, Englewood Cliffs, N. J., 1971. Zbl 0212.36601 MR 0276251621

[11] A. Heller and I Reiner, Indecomposable representations. Illinois J. Math. 5 (1961), 314-323. Zbl 0111.03101 MR 0122890623

[12] P. M. Morse and H. Feshbach, Methods of theoretical physics. Part I. Internat. Series Pure Appl. Phys., McGraw-Hill, New York 1953. Zbl 0051.40603 MR 0059774647

[13] A. Suslin, E. Friedlander, and C. Bendel, Support varieties for infinitesimal group schemes. J. Amer. Math. Soc. 10 (1997), 729-759. Zbl 0960.14024 MR 1443547612

Received March 1, 2009

Jon F. Carlson, Department of Mathematics, University of Georgia, Athens, GA, U.S.A.

E-mail: jfc@math.uga.edu

Eric M. Friedlander, Department of Mathematics, University of Southern California, Los Angeles, CA, U.S.A.

E-mail: ericmf@usc.edu

Andrei Suslin, Department of Mathematics, Northwestern University, Evanston, IL, U.S.A.

E-mail: suslin@math.northwestern.edu 